\title{
Annonaceae: Breaking the Wall of Inflammation
}

\author{
Ali Attiq, Juriyati Jalil* and Khairana Husain \\ Drug and Herbal Research Centre, Faculty of Pharmacy, Universiti Kebangsaan Malaysia, Kuala Lumpur, Malaysia
}

\section{OPEN ACCESS}

Edited by:

Anna Karolina Kiss,

Medical University of Warsaw, Poland

Reviewed by:

Subhalakshmi Ghosh, Jadavpur University, India

Raghuram Kandimalla,

Institute of Advanced Study in Science

and Technology, India

Krystyna Skalicka-Wozniak,

Medical University of Lublin, Poland

*Correspondence:

Juriyati Jali

juriyatijaliıukm.edu.my

Specialty section: This article was submitted to

Ethnopharmacology,

a section of the journal

Frontiers in Pharmacology

Received: 31 May 2017 Accepted: 03 October 2017

Published: 20 October 2017

Citation:

Attiq A, Jalil J and Husain K (2017) Annonaceae: Breaking the Wall of Inflammation.

Front. Pharmacol. 8:752. doi: 10.3389/fphar.2017.00752
Inventories of tropical forests have listed Annonaceae as one of the most diverse plant families. For centuries, it is employed in traditional medicines to cure various pathological conditions including snakebite, analgesic, astringent, diarrhea, dysentery, arthritis pain, rheumatism, neuralgia, and weight loss etc. Phytochemical analysis of Annonaceae family have reported the occurrence of alkaloids, flavonoids, triterpenes, diterpenes and diterpene flavone glycosides, sterols, lignans, and annonaceous acetogenin characteristically affiliated with Annonaceae sp. Numerous past studies have underlined the pleotropic pharmacological activities of the crude extracts and isolated compounds from Annonaceae species. This review is an effort to abridge the ethnobotany, morphology, phytochemistry, toxicity, and particularly focusing on the anti-inflammatory activity of the Annonaceae species.

Keywords: anti-inflammatory, annonaceae, cytokines, prostaglandins, nuclear factor kappaB, reactive oxygen species, cycloxegenase

\section{INTRODUCTION}

Inflammation is a human body's defense mechanism that can be triggered by numerous factors including physical trauma, exposure to allergen, chemical or heat stimulus, and microbial infection (Kidd and Urban, 2001; Guo et al., 2015) The inflammation is a tightly regulated process involving pro-inflammatory stimulus, to initiate and maintain inflammation and anti-inflammatory signals which helps in shutting down the process (Dinarello, 1997; Ziebell and Morganti-Kossmann, 2010). However, there are several internal or external factors that can disturb this regulatory network of pro and anti-inflammatory mediators (Cytokines, chemokine etc.), and hence giving rise to various inflammatory conditions including inflammatory bowel disease rheumatoid arthritis, multiple sclerosis, and chronic asthma (Sahlmann and Ströbel, 2016). Several therapeutic options like nonsteroidal inflammatory drugs (NSAIDs), corticosteroids and Disease Modifying Anti Rheumatic Drugs (DMARDs) are now available to treat these life threatening and painful inflammatory conditions (Hoes et al., 2010; Weber and Noels, 2011). However, prolong treatment with these drugs have been associated with serious and sometimes life threatening side effects including uncontrolled hypertension, gastric ulcers, acute kidney failure, glaucoma, heart failure etc., (Jachak, 2006; Huscher et al., 2009).

Natural products have been the counter stone for the traditional medicine practices around the world for centuries. Throughout this time numerous plant species have been used in the form of tinctures, decoctions, and dried powder form to treat all sort of inflammatory condition (Van Wyk and Wink, 2004). The knowledge of medicinal activity has been gathered over the course of centuries, using personal observations and trial and error methods. Hence it is imperative to carry out further research on plants species with medicinal properties to validate their therapeutic activity. During the last century, natural products have proved to be an essential pipeline for 
drug discovery and drug design (Al-Dhubiab, 2012) and had gain great significance due to their extraordinary value in the field of pharmacology and therapeutics (Kumar and Khanum, 2012; Moghadamtousi et al., 2013; Akaberi et al., 2015).

Annonaceae has been listed among the most diversified families of tropical forest due to its heterogeneity and abundance in this region (Phillips and Miller, 2002; Couvreur et al., 2011). This review is an effort to highlight the botanical features, phytochemistry, medicinal uses, and anti-inflammatory activities and toxicity of most notable species of Annonaceae. Moreover, this review has analyzed the scientific data from experimental studies to validate the traditional uses and claimed anti-inflammatory activities of few Annonaceae species.

\section{CLASSIFICATION AND BOTANICAL FEATURES OF ANNONACEAE SPECIES}

The botanical features of the Annonaceae family can vary from species to species depending upon its origin, climate, and topography. Its botanical diversity can range from trees to shrubs, evergreen climbers, with elongated cylindrical-shaped intracellular resin channels and broad and well-developed septate pith in the stems (Hamonnière et al., 1977). The aromatic flowers bloom before they are completely developed; they are axillary, singular or grouped, hermaphrodite, and regular in shape. The stamens are typically considerable in numbers, hypogenous, and spirally arranged. Fruits are made up of clusters of berries and that are widely consumed in tropical regions due to their high nutritional value. Seeds are usually enlarged and have irregular surfaced endosperm with a small embryo (Hutchinson, 1959; Hamonnière et al., 1977; Takhtajan, 2009).

For over a considerable period of time botanists have confused and mistakenly included monotypic Eupomatiaceae in Annonaceae family, but the differential botanical features of Annonaceae have made it easier to differentiate Annonaceae from Eupomatiaceae. For instance the presence of vessel perforations of leaves, broad and high multi seriate xylem rays which give a cross-sectional "cobweb-like" wood structure are characteristically associated with Annonaceae species (Koek-Noorman and Westra, 2012). Moreover, the distichous phyllotaxis leaves, petals, sepals, and stamens are in the sets of three (trimerous) (Watson and Dallwitz, 1999). While calyx and corolla, long chalaza are in a perfect symmetrical ovule (Sauquet and Le Thomas, 2003; Sauquet et al., 2003).

The first classification of Annonaceae family was carried out by Dunal (1817). The classification of Dunal was solely based on fruit morphology. Later, Baillon (1868) and Diels and Alder (1932) used flower characters to develop a new classification of Annonaceae family. However, the classification of Fries (1959) was comprehensive and most authentic. He improvised the pervious classifications by combining floral characteristics and fruit morphology and hence these interventions made the classification of Fries to be a gold stranded for future taxonomical studies as well (Zomlefer, 1994).

The presence of many primitive and archaic morphological features and their ability to survive the mass extinctions has characterized the Annonaceae species as "living fossils." The order Magnoliales is among the largest orders, comprising six families including Eupomatiaceae, Myristicaceae, Magnoliaceae, Degeneriaceae, Himantandraceae, and Annonaceae (Davis and Wurdack, 2004; Soltis and Soltis, 2004). With 180 genra with more than three thousand species, Annonaceae family is the largest family of this order. Annonaceae species are predominantly distributed in parts of world with abundant rainfall including Malaysia, Indonesia, Japan, India, Sri Lanka, and Pakistan.

\section{TRADITIONAL USES}

Natural products have been the basis of many traditional medicines from all around the world. Moreover, these medicinal practices have played a significant role in the providing the remedies for all sorts of inflammatory conditions. Annonaceae family is very famous in tropical regions due to its widespread use in traditional medicine For instance, the juice of the macerated leave of Annona muricata is used in Brazil for arthritis, rheumatism, and neuralgia (Cercato et al., 2015). Moreover, in some parts of Indonesia the dried leave are orally ingested for its potent analgesic effect (Badrie and Schauss, 2010; Bele et al., 2011). Moreover, leaves of Anaxagorea dolichocarpus, commonly known Jari Jari has been a traditional remedy of articular rheumatisms for centuries. Native South African tribes tropically apply the gridded Jai Jari leaves with castor oil to treat articular rheumatisms (DeFilipps et al., 2004). In Brazil, fresh fruit of Annona dioica is used due to its long history of wound healing in fresh wounds (Formagio et al., 2013a). Likewise in Amazona, the northwestern part of Brazil Duguetia chrysocarpa leaves and twigs are ground together and extract of this mixture is used as a remedy for inflammatory bowl diseases and gastrointestinal ulcers (Almeida et al., 2011, 2012). While, in other parts of the world have benefited from the seed, leave and fruits of Annona reticulata. Counties like West Indies and Dominican Republic use fruit decoction as a traditional remedy for bronchitis. While, oral ingestion of the powdered leaves are reported to decease the frequency and intensity of asthma attack (Auddy et al., 2003; Bhalke and Chavan, 2011). Peninsular Malaysian rain forests are famous for their ecological diversity and due to this heterogeneity in plant species it has been origin of numerous household remedies for inflammatory diseases. For instance, Cananga odorata commonly known as perfume tree is very famous in this region due to its strong anti-pyretic and anti-inflammatory activity (Duke and Beckstrom-Sternberg, 2000). Fresh wounds are wash with bark extract and decoction to reduce inflammation and facilitates the wound healing (Tan et al., 2015). While, the extract is poured into the eye in order to reduce the ophthalmic inflammation (Scartezzini and Speroni, 2000; Rahman et al., 2005). Likewise, in Sabah and Sarawak, western part of the Malaysia several Annonacaeae species including Enicosanthellum pulchrum, Friesodielsia latifolia, Uvaria grandi, Uvaria rufa are in used a remedy for pedal edema (Nordin et al., 2014), generalized body pain (Araujo et al., 2017), antipyretic (Parmar et al., 1994), and anti-inflammatory (Buncharoen et al., 2016). 
Moreover, in Africa $80 \%$ of the population is still dependent on the traditional remedies due to easy accessibility and low cost. Several indigenous species including Enantia chlorantha have been reported to possess strong immunomodulatory activity. The Powdered bark is mixed with citrus lemon and then paste is used as dressing for artharitis (Tan et al., 2000). While, in Sudan ethanolic root extract and whole fruit of Xylopia aethiopica is used as a remedy for neuralgia, headache and colic pain (Ogunkunle and Ladejobi, 2006; Woode et al., 2012). While in central Africa the root decoction of Xylopia parvifolia is as a natural remedy for pain management and roots are chews and then swallowed for gastrointestinal ulcers and inflammations. The traditional uses of most widely used Annonaceae species are summarized in Table 1 along with the parts used and mode of administration.

\section{ANTI-INFLAMMATORY ACTIVITY}

Portfolios of tropical forests perpetually list Annonaceae as one of the most diverse plant families (Phillips and Miller, 2002). With respect to the species abundance it contributes notably to the diversity of trees in Neotropical region (Saunders, 2012). Similarly, this diversity can also be seen in its wide range of phyto-constituents. Phytochemical studies of this family have reported the presence of alkaloid (Malebo et al., 2013; Kouam et al., 2014; Soares et al., 2015), cyclopeptides (Wu P. et al., 2014; Moghadamtousi et al., 2015), flavonoids (Lage et al., 2014; Chokchaisiri et al., 2015), terpenoids (Annan et al., 2013; Rabelo et al., 2016), and lignans (Moreira et al., 2013; Nguyen et al., 2015; Rayanil et al., 2016). Several bioactivities including antimalarial (Frausin et al., 2014; Meira et al., 2015), antiplatelet, (Thang et al., 2013a; González-Esquinca et al., 2014), and antiinflammatory activity (Kandimalla et al., 2016) are just to name a few. Cytotoxicity of Annonaceous acetogenins has made this family of major interest for novel anti-cancer drugs (Han et al., 2015; Yang et al., 2015). However, the focus of this study is to demonstrate vast array potential compounds, for future drug discovery with anti-inflammatory activity. Annonaceae species have grave importance with relevance to its wide range of antiinflammatory activities. In vitro and in vivo studies including isolated compounds as well as rudimentary crude extracts have shown potent activity in all sorts of inflammation. An in depth detail of Annonaceae species has been summarize in Table 2.

\section{Nuclear Factor Kappa B (NF-кB) Inhibition}

$\mathrm{NF}-\kappa \mathrm{B}$ is a principal transcription factor involved in regulating the gene expression of more than thousand regulatory proteins, including certain pro-inflammatory cytokines. These cytokines further induce the transcription of adhesion molecules, critical in leukocyte infiltration and transmigration to the site of injury and inflammation (Barnes and Karin, 1997; Tak and Firestein, 2001; Akira et al., 2006). Early studies on NF-кB has confirmed its role in gene regulation of the Igк light chain and numerous other regulatory genes responsible for carrying out normal physiological functions, including immune modulation, acute inflammatory response, cell differentiation and apoptosis (Baeuerle and Baltimore, 1996; Yamamoto and
Gaynor, 2001a; Karin et al., 2006). Five mammalian proteins have been recognized in NF- $\mathrm{B}$ activation pathway: NF- $\kappa \mathrm{B}$ (p50 and its precursor p105), NF-кB2 (p52 and its precursor p100), p65 (RelA), RelB, and c-Rel. These proteins can interact with each other as homo- or heterodimers, depending upon their active or latent state. A Rel homology domain is characteristically present on all the NF- $\kappa \mathrm{B}$ members, which encompasses a nuclear localization array for binding of specific DNA sequences, sites for dimerization, and interface with inhibitory I $\mathrm{B}$ proteins. In the cytoplasm, these inhibitory proteins (I $\mathrm{B} \alpha, \mathrm{I} \kappa \mathrm{B} \beta, \mathrm{I} \kappa \mathrm{B} \varepsilon$, and $\mathrm{Bcl}-3$ ) are non-covalently bonded to the NF- $\kappa \mathrm{B}$ dimer keeping it in an inactive state. Upon receiving the stimulus, $26 \mathrm{~S}$ proteosome seeks the help of IкB kinases for the phosphorylation and polyubiquition of IкB (Karin and Ben-Neriah, 2000; Broide et al., 2005). This makes the way for promoter genes to interact with nuclear localization site and activates the transcriptional factors. This activation permits NF- $\kappa \mathrm{B}$ to be translocated into the nucleus, followed by the transcription of numerous cytokines pro-inflammatory cytokines (IL-1, IL-2, IL-6, IL-12, TNF $\alpha$ ), chemokines (IL8, Rantes, MCP-1, MIP-1 $\alpha$, eotaxin), cell adhesion molecules (ICAM-1 and VCAM-1) acute phase proteins (SAA, CRP), and the inducible enzymes, nitric oxide synthetase (iNOS) and cyclooxygenase (COX-2) (Barnes and Karin, 1997; Tak and Firestein, 2001; Yamamoto and Gaynor, 2001a; Gordon and Taylor, 2005). Henceforth, NF- $\mathrm{B}$ activation can be inculpated for the production of numerous immunomudulators, responsible for many inflammatory diseases. Clinical data has suggested that elevated expression of these inflammatory mediators have been observed in inflamed synovial tissues, hence leading to the development and progression of rheumatoid arthritis (Marok et al., 1996). Moreover, overexpressed COX-2 expression contributes to the prostanoids production, which further promotes the synthesis of IL-1 and TNF $\alpha$, leading to chronic inflammatory diseases. Thus, the natural product with NF-кB inhibitory activity represents a potential therapeutic alternative in treating inflammation (Barnes and Karin, 1997; Yamamoto and Gaynor, 2001b).

Numerous Annonaceae species have been investigated for the inhibitory activity on NF-kB pathway. For example, styryl lactones from the genus Goniothalamus are secondary metabolites with either 5-or 6-membered lactones (De Fátima et al., 2006) with several reported bioactivities including cytotoxicity, apoptosis, and anti-inflammatory (De Fátima et al., 2006; Kuo et al., 2011). In recent work of Orlikova et al. (2013), goniothalamin (1) (GTN) (Figure 1) a styryl-lactone isolated from the Goniothalamus macrophyllus was evaluated on TNF- $\alpha$ induced NF- $\kappa \mathrm{B}$ activation. GTN inhibited the TNF- $\alpha$ induced $\mathrm{NF}-\kappa \mathrm{B}$ activation in K562 chronic myelogenous leukemia cells at a concentration of $5 \mu \mathrm{M}$. Moreover, GTN also prevented NF$\kappa \mathrm{B}$ binding with its DNA transcription factors. Translocation of the p50/p65 heterodimer to the nucleus was down regulated and TNF- $\alpha$ activated interleukin 8 (IL-8) expression was also significantly reduced. Furthermore, in a phytochemical evaluation of Indonesian plant species, methanolic extract of Alphonsea javanica showed potent anti-inflammatory activity by inhibiting NF- $\kappa$ B activation in raw macrophage 264.7 cells 
TABLE 1 | Medicinal uses of most commonly used Annonaceae species.

\begin{tabular}{|c|c|c|c|c|c|c|}
\hline Plant name & Country region & $\begin{array}{l}\text { Local name/Common } \\
\text { name }\end{array}$ & Medicinal uses & Part (used) & $\begin{array}{l}\text { Mode of } \\
\text { usage/preparation }\end{array}$ & References \\
\hline $\begin{array}{l}\text { Alphonsea javanica } \\
\text { Scheff. }\end{array}$ & Indonesia & Aku Battu & $\begin{array}{l}\text { Rheumatism and } \\
\text { edema }\end{array}$ & Leave & Ethanolic extract & Johnson et al., 2013 \\
\hline $\begin{array}{l}\text { Annona crassiflora } \\
\text { Mart. }\end{array}$ & Cerrado biome & $\begin{array}{l}\text { Araticum of the Cerrado, } \\
\text { marolo, or panã }\end{array}$ & $\begin{array}{l}\text { Rheumatism, wounds } \\
\text { healing, healing, and } \\
\text { anti-inflammatory }\end{array}$ & Fruit & No information & $\begin{array}{l}\text { Vilar et al., 2008; Silva } \\
\text { et al., } 2014\end{array}$ \\
\hline Annona dioica St. Hill & Brazil & Ceraticum and ariticum & Rheumatism & Fruits and leaves & $\begin{array}{l}\text { Dried leave paste and } \\
\text { fresh fruit decoction }\end{array}$ & Formagio et al., 2013a \\
\hline Annona muricata Linn & Brazil & $\begin{array}{l}\text { Araticum, condessa, } \\
\text { graviola }\end{array}$ & $\begin{array}{l}\text { Analgesic, Arthritis } \\
\text { pain, rheumatism, } \\
\text { neuralgia }\end{array}$ & Fruit Leaf & $\begin{array}{l}\text { Juice of fresh fruit } \\
\text { and water extract of } \\
\text { leaves ( } 12 \mathrm{~h} \text { of cold } \\
\text { maceration) }\end{array}$ & $\begin{array}{l}\text { Badrie and Schauss, } \\
2010 \text {; Cercato et al., } \\
2015\end{array}$ \\
\hline Annona reticulata Linn & West Indies & Ramphal & $\begin{array}{l}\text { Bronchitis, Asthma, } \\
\text { Bowel inflammation }\end{array}$ & $\begin{array}{l}\text { fruit } \\
\text { Seeds } \\
\text { Leaf }\end{array}$ & $\begin{array}{l}\text { Oral ingestion of the } \\
\text { leaf powder } \\
\text { Decoction of fruit in } \\
\text { boiled water. } \\
\text { Powdered seeds } \\
\text { ingested }\end{array}$ & $\begin{array}{l}\text { Auddy et al., 2003; } \\
\text { Bhalke and Chavan, } \\
2011\end{array}$ \\
\hline $\begin{array}{l}\text { Annona senegalensis } \\
\text { Persoon }\end{array}$ & Nigeria & Ukopko (Idoma) & $\begin{array}{l}\text { Anti-inflammatory and } \\
\text { analgesic }\end{array}$ & $\begin{array}{l}\text { Leaf } \\
\text { Root }\end{array}$ & $\begin{array}{l}\text { Roots and bark are } \\
\text { ground together and } \\
\text { their Decoction is } \\
\text { used }\end{array}$ & Ofukwu et al., 2008 \\
\hline $\begin{array}{l}\text { Annona vepretorum } \\
\text { Mart }\end{array}$ & Brazil & Araticum, bruteira & $\begin{array}{l}\text { Analgesic and } \\
\text { anti-inflammatory }\end{array}$ & Leave & $\begin{array}{l}\text { Methanolic Leaf } \\
\text { extract }\end{array}$ & Silva et al., 2015 \\
\hline $\begin{array}{l}\text { Cananga odorata } \\
\text { Hook.f. and Thomson }\end{array}$ & Malaysia and India & $\begin{array}{l}\text { Kenanga utan, perfume } \\
\text { tree, sananga oil, kenanga } \\
\text { wood }\end{array}$ & $\begin{array}{l}\text { Rheumatism } \\
\text { Ophthalmic } \\
\text { inflammation and } \\
\text { Wound healing }\end{array}$ & Bark & $\begin{array}{l}\text { Decoction is used to } \\
\text { wash fresh wounds } \\
\text { and extract dropped } \\
\text { into eyes for } \\
\text { inflammation }\end{array}$ & $\begin{array}{l}\text { Duke, 2000; Scartezzini } \\
\text { and Speroni, 2000; } \\
\text { Rahman et al., 2005 }\end{array}$ \\
\hline $\begin{array}{l}\text { Duguetia chrysocarpa } \\
\text { Maas }\end{array}$ & Brazil & Pindaíba-da-mata & $\begin{array}{l}\text { Rheumatism and Bowl } \\
\text { inflammation }\end{array}$ & Leave and twigs & $\begin{array}{l}\text { Powdered leaves and } \\
\text { twigs extract is drunk } \\
\text { to relive inflammatory } \\
\text { conditions }\end{array}$ & $\begin{array}{l}\text { Almeida et al., 2011, } \\
2012\end{array}$ \\
\hline $\begin{array}{l}\text { Enantia chlorantha var. } \\
\text { soyauxii Engler and } \\
\text { Diels }\end{array}$ & Africa & African yellow wood & $\begin{array}{l}\text { Arthritis and wound } \\
\text { healing }\end{array}$ & Bark & $\begin{array}{l}\text { Powdered bark with } \\
\text { citrus lemon used as } \\
\text { dressing }\end{array}$ & Tan et al., 2000 \\
\hline $\begin{array}{l}\text { Enicosanthellum } \\
\text { pulchrum King) } \\
\text { Heusden }\end{array}$ & Malaysia & Disepalum & $\begin{array}{l}\text { Rheumatism fever, } \\
\text { asthma, and edema }\end{array}$ & Leave & $\begin{array}{l}\text { Fine powder can } \\
\text { ingested directly or } \\
\text { water decocotion can } \\
\text { also be used for } \\
\text { asthma and } \\
\text { rheumatism }\end{array}$ & Nordin et al., 2014 \\
\hline $\begin{array}{l}\text { Fissistigma oldhamii } \\
\text { (Hemsl.) Merr }\end{array}$ & Southern China & Oldhamii & Rheumatoid arthritis & Stems and roots & $\begin{array}{l}\text { Dried stem and root } \\
\text { powder is orally } \\
\text { ingested }\end{array}$ & Araujo et al., 2017 \\
\hline $\begin{array}{l}\text { Friesodielsia latifolia } \\
\text { Hook.f. and Thomson }\end{array}$ & Malaysia & No information & $\begin{array}{l}\text { Gernalized body pain } \\
\text { and Pedal edema }\end{array}$ & Roots & Root decoction & Wiart, 2007 \\
\hline $\begin{array}{l}\text { Mitrella Kentii (Blume) } \\
\text { Miq }\end{array}$ & Indonesia & Kiawi & $\begin{array}{l}\text { Antipyretics and } \\
\text { Edema }\end{array}$ & Roots & $\begin{array}{l}\text { Decoction to treat } \\
\text { fever }\end{array}$ & Wiart, 2006 \\
\hline $\begin{array}{l}\text { Monodora myristica } \\
\text { (Gaertn.) Dunal }\end{array}$ & Ivory coast & M Kpo. Abidjan district & $\begin{array}{l}\text { Febrile pains, eye } \\
\text { diseases and } \\
\text { hemorrhoids and } \\
\text { headaches }\end{array}$ & Fruits Seed & $\begin{array}{l}\text { Seeds and fruits } \\
\text { consumed in whole } \\
\text { or ground to be used } \\
\text { in soup and strewed }\end{array}$ & Moukette et al., 2015 \\
\hline $\begin{array}{l}\text { Polyalthia longifolia cv. } \\
\text { Pendula }\end{array}$ & India & Ashoka & Fever & Bark & Bark decoction & $\begin{array}{l}\text { Chadha, 1985; Katkar } \\
\text { et al., } 2010\end{array}$ \\
\hline $\begin{array}{l}\text { Uvaria grandiflora } \\
\text { Roxb. ex Hornem }\end{array}$ & Malaysia & Pisang Tandok & $\begin{array}{l}\text { Wound healing, Fever } \\
\text { with chills and rigors }\end{array}$ & Leaves & $\begin{array}{l}\text { Paste of the leaves } \\
\text { wrapped around the } \\
\text { abdomen part of } \\
\text { children }\end{array}$ & Parmar et al., 1994 \\
\hline
\end{tabular}


TABLE 1 | Continued

\begin{tabular}{|c|c|c|c|c|c|c|}
\hline Plant name & Country region & $\begin{array}{l}\text { Local name/Common } \\
\text { name }\end{array}$ & Medicinal uses & Part (used) & $\begin{array}{l}\text { Mode of } \\
\text { usage/preparation }\end{array}$ & References \\
\hline Uvaria rufa Blume & Malaysia & Larak or Pisang-pisang & $\begin{array}{l}\text { Antipyretic and } \\
\text { anti-inflammatory }\end{array}$ & $\begin{array}{l}\text { Leave } \\
\text { Bark }\end{array}$ & $\begin{array}{l}\text { Soaked leaves in } \\
\text { water } \\
50 \% \text { ethanolic extract } \\
\text { of bark }\end{array}$ & $\begin{array}{l}\text { Buncharoen et al., } \\
2016\end{array}$ \\
\hline $\begin{array}{l}\text { Xylopia aethiopica } \\
\text { (Dunal) A.Ric }\end{array}$ & Sudan & Ethiopia or Negro pepper & $\begin{array}{l}\text { Rheumatism, } \\
\text { headache, colic pain, } \\
\text { and neuralgia }\end{array}$ & $\begin{array}{l}\text { Fruits } \\
\text { Seeds }\end{array}$ & $\begin{array}{l}\text { Ethanolic fruit extract } \\
\text { and The dried fruits } \\
\text { are used as whole }\end{array}$ & $\begin{array}{l}\text { Ogunkunle and } \\
\text { Ladejobi, } 2006 \text {; Woode } \\
\text { et al., } 2012\end{array}$ \\
\hline $\begin{array}{l}\text { Xylopia aromatic Lam. } \\
\text { Mart }\end{array}$ & Columbia an brazil & Monkey pepper & $\begin{array}{l}\text { Pulmonary } \\
\text { inflammation and } \\
\text { hemorrhoids }\end{array}$ & $\begin{array}{l}\text { Roots } \\
\text { Leaves }\end{array}$ & $\begin{array}{l}\text { Insertion of root } \\
\text { pieces into rectum } \\
\text { and leaves burnt and } \\
\text { smoke inhaled }\end{array}$ & Woguem et al., 2014 \\
\hline $\begin{array}{l}\text { Xylopia parvifolia } \\
\text { Hook.f. and Thomson }\end{array}$ & $\begin{array}{l}\text { East and Central } \\
\text { Africa, India }\end{array}$ & Netawu/Athu ketiya & $\begin{array}{l}\text { Gastrointestinal ulcers } \\
\text { and analgesic }\end{array}$ & Roots & $\begin{array}{l}\text { Decoction Finely } \\
\text { drinded powder }\end{array}$ & Nishiyama et al., 2006 \\
\hline
\end{tabular}

(Johnson et al., 2013). Moreover, I $\mathrm{B} \alpha$ phosphorylation was also significantly reduced. This phytochemical analysis suggested that reported activity was due to the presence of an styryl lactone, altholactone (2) (Figure 1). However, according to Taha et al. (2014) methanolic bark extract of Pseuduvaria monticola have shown insignificant results in anti-diabetic evaluation studies. Other parameters including, NF- $\kappa \mathrm{B}$ translocation were also evaluated on pancreatic insulinoma cells of mice. Test extract failed to show inhibitory activity against TNF induced NF-кB translocation. However, Shih et al. (2010) reported a completely different anti-inflammatory activity of Polyalthia longifolia. Since microglia mediated inflammation is involved in the pathway responsible for neuronal cell death in neurodegenerative diseases (Gebicke-Haerter, 2001). Hence, this study was carried out to evaluate the effects of $P$. longifolia var. pendul isolate, 6hydroxycleroda-3,13-dien-15,16- olide (3)(PL3) (Figure 1) on LPS induced microglial inflammation. PL3 (3) successfully decreased the cell viability in neuroblastoma SH-SY5Y cells. Subsequently, it decreased the activity of $\mathrm{NF}-\kappa \mathrm{B}$ and the degradation of $\mathrm{I} \kappa \mathrm{B} \alpha$. PL3(3) also boosted HO-1 expression, which is a known cytoprotective and anti-inflammatory enzyme. Moreover, microglial activation is also associated with the complete or partial loss of dopaminergic neurons in Parkinson's disease (PD) (McGeer and McGeer, 2004; Suzumura et al., 2006; Block et al., 2007). Hence, if early measures are taken to suppress the microglial activation, then it would be an important step in suppressing the progression of PD. $\alpha$-asarone (4) (Figure 1), an active constituent found in few Annonaceae species, has proved to beneficial in the early treatment of PD (López et al., 1993; Silva et al., 2007; Kim et al., 2015). Kim et al. (2015) reported that $\alpha$-asarone(4) decreased the pro- inflammatory cytokine production in LPS induced BV-2 cells. The detailed mechanistic study revealed that reported activity of $\alpha$-asarone was due to the inhibition of NF- $\kappa \mathrm{B}$, by blocking degradation of Ik-B signaling in BV-2 microglial cells. Moreover, in a recent study it was suggested that methanolic extract of $A$. reticulata have shown significant neuro-protective response in $\mathrm{H}_{2} \mathrm{O}_{2}$ induced neuronal damage in SHSY5Y cells and DRG neuronal cells. After the completion of the drug treatment the levels of pro-inflammatory cytokines, iNOS, and NF- $\mathrm{B}$ activation was significantly reduced in a dose dependent manner. Hence it was concluded from this study methanolic extract of $A$. reticulata has potential to inhibit neuronal inflammation, neurogenic pain, and oxidative stress by inhibiting NF- $\mathrm{B}$ inflammatory pathway (Kandimalla et al., 2017).

NF- $\kappa \mathrm{B}$ activation is accountable for the development and progression of solid and hemopoietic malignancies (Van Waes, 2007; Yang F. et al., 2012). Therefore, NF- $\mathrm{B}$ is often referred as an oncogene as well. Although, NF- $\mathrm{B}$ activation is not a prerequisite for tumor, but it plays an important role in moderating inflammation, setting up the tumor microenvironment and stimulating immunomodulatory cells including, pro and antiinflammatory cytokines, and chemokine production (Mantovani et al., 2008, 2010; Grivennikov et al., 2010). Here, two pathological conditions have been stated as examples to signify the role of NF- $\mathrm{B}$ activation in inflammation leading to cancer. The first example is Colitis-associated colon cancer (CAC) a classical inflammation-driven cancer and, secondly, hepatitis $\mathrm{C}$ induced hepatic cancer is the most prominent examples of inflammation leading to cancer (Greten et al., 2004; Pikarsky et al., 2004). Henceforth, the agents with the tenacity to subdue the NF- $\mathrm{B}$ translocation are of major interest due to their dual action on inflammation and cancer development (Zhang et al., 2005; Opferman, 2008; Cheng et al., 2012). Moghadamtousi et al. (2014a) evaluated the ethyl acetic acid extract of A. muricata (leaves) (AMEAE) on A549 lung cancer cells. Cell viability study demonstrated the specific cytotoxic impact of AMEAE toward A549, with an $\mathrm{IC}_{50}$ of $5.09 \pm 0.41 \mu \mathrm{g} / \mathrm{mL}$ after $72 \mathrm{~h}$ of treatment. In addition, AMEAE also inhibited the translocation of NF-кB from the cytoplasm to nucleus. Apropos to last study reported, Pieme et al. (2014) suggested that the presences of phenolic compounds are the key elements for the reported NF- $\kappa \mathrm{B}$ activity of A. muricata (Duraipandiyan et al., 2006; Jiménez et al., 2014; Solomon-Wisdom et al., 2014).

\section{Prostaglandin (PGs) Inhibition}

The association between the PGs and inflammation was acknowledged in 1971. While two research groups reported 
TABLE 2 | Mechanism of action of extracts and isolates of Annonacae species with potent anti-inflammatory activity.

\begin{tabular}{|c|c|c|c|c|c|}
\hline Plant name & Part used & Isolate compound/Extract & Class & Mechanism of action/Conclusion & References \\
\hline Alphonsea javanica Scheff & Leaf & $\begin{array}{l}(+) \text {-Altholactone and } \\
(+) \text {-goniothalmin }\end{array}$ & Styryl-lactone & $\begin{array}{l}\text { LPS induced NO production, IKB- } \alpha \text {, and } \\
\text { expression of } \mathrm{iNOS} \text { and COX- } 2 \text { was } \\
\text { significantly reduced in Raw macrophage } \\
264.7 \text { cells at } \mathrm{IC}_{50} \text { range of } 0.8-5.0 \mu \mathrm{M}\end{array}$ & $\begin{array}{l}\text { Johnson } \\
\text { et al., } 2013\end{array}$ \\
\hline Annona crassiflora Mart & Leave & Methanolic extract & - & $\begin{array}{l}\text { Oral treatment with } 100 \text { and } 300 \mathrm{mg} / \mathrm{kg} \\
\text { reduced carrageenan-induced edeme by } 53 \pm \\
7 \text { and } 47 \pm 10 \% \text { and leukocyte migration was } \\
\text { suppressed by } 60 \pm 7 \text { and } 63 \pm 7 \% \text {, } \\
\text { respectively }\end{array}$ & $\begin{array}{l}\text { Rocha et al., } \\
2016\end{array}$ \\
\hline Annona Cheromola Mill & Fruit & $\begin{array}{l}\text { Ethanol, methanol and dimethyl } \\
\text { formammide }\end{array}$ & - & $\begin{array}{l}\text { Methanolic extracts has shown maximum } \\
\text { Extract has exhibited potent radical scavenging } \\
\text { activity toward 1,1-Diphenyl-2-picryl-hydrazyl } \\
\text { and Superoxide anion at } I_{50} \text { range of } \\
100-250 \mu \mathrm{g} / \mathrm{mL}\end{array}$ & $\begin{array}{l}\text { Barreca et al., } \\
2011\end{array}$ \\
\hline Annona dioica A.St.-Hil & Leaves & Quercetin and kaempferol & Flavonoids & $\begin{array}{l}\text { Leukocytes migration activity was inhibited at } \\
\mathrm{IC}_{50} \text { Value of } 8.53 \text { and } 10.57 \mu \mathrm{g} / \mathrm{mL} \text {, } \\
\text { respectively }\end{array}$ & $\begin{array}{l}\text { Formagio } \\
\text { et al., } \\
2013 a, b\end{array}$ \\
\hline \multirow[t]{2}{*}{ Annona glabra L } & Fruit & Isodesacetyluvaricin & Acetogenins & $\begin{array}{l}\text { Selectively inhibited COX-2 and mRNA } \\
\text { expression at dose } 5 \mu \mathrm{M}\end{array}$ & $\begin{array}{l}\text { Wu et al., } \\
2012\end{array}$ \\
\hline & Fruits & $\begin{array}{l}\text { 7ß,17-dihydroxy-ent-kaur-15-en- } \\
\text { 19-oic acid } \\
\text { 19-O- } \beta \text {-d-glucopyranoside ester }\end{array}$ & Ent-kaurane diterpenoids & $\begin{array}{l}\text { Significant inhibition in iNOS production was } \\
\text { observed with an } \mathrm{IC}_{50} \text { of } 0.01 \mu \mathrm{M}\end{array}$ & $\begin{array}{l}\text { Nhiem et al., } \\
2015\end{array}$ \\
\hline Annona montana Macfed & Seeds & Cyclomontanins & Cyclopeptides & $\begin{array}{l}\text { Significant inhibition in TNF- } \alpha \text { and IL- } 6 \\
\text { production was observed in Murine } \\
\text { macrophage J774A with an IC50 value of } \\
30 \mu \mathrm{g} / \mathrm{mL}\end{array}$ & $\begin{array}{l}\text { Chuang et al., } \\
2008\end{array}$ \\
\hline \multirow[t]{5}{*}{ Annona muricata L } & Leaves & Ethanolic extract & - & $\begin{array}{l}\text { Reduced the number of abdominal contortions } \\
\text { by } 14.42 \% \text { at } 200 \mathrm{mg} / \mathrm{Kg} \text {, increased the } \\
\text { reaction time on a hot plate at doses of } 200 \\
\mathrm{mg} / \mathrm{kg} \text { and carrageenan induced paw edema } \\
\text { was reduced by } 29.33 \% \text { at } 200 \mathrm{mg} / \mathrm{kg}\end{array}$ & $\begin{array}{l}\text { de Sousa } \\
\text { et al., } 2010\end{array}$ \\
\hline & Unripe fruit & Lyophilized fruit extract & - & $\begin{array}{l}\text { Infiltrations of inflammatory meditators were } \\
\text { significantly inhibited with pretreatment of } 100 \\
\mathrm{mg} / \mathrm{mL} \text { extract in mice }\end{array}$ & $\begin{array}{l}\text { Ishola et al., } \\
2014\end{array}$ \\
\hline & & & & $\begin{array}{l}\text { Reduction in ROS and } \mathrm{PGE}_{2} \text {.production was } \\
\text { observed at dose of } 200 \text { and } 400 \mathrm{mg} / \mathrm{kg} \text { in } \\
\text { mice, respectively }\end{array}$ & $\begin{array}{l}\text { Moghadamtous } \\
\text { et al., } 2014 \mathrm{~b}\end{array}$ \\
\hline & Leaf & Ethanolic extract & - & $\begin{array}{l}\text { Significantly decreased of TNF } \alpha \text { and IL-1 } \beta \\
\text { levels were reported in Freund's adjuvant } \\
\text { induced arthritis mice at dose of } 100 \mathrm{mg} / \mathrm{kg}\end{array}$ & $\begin{array}{l}\text { Foong and } \\
\text { Hamid, } 2012\end{array}$ \\
\hline & Leave & Aqueous Extract & - & $\begin{array}{l}\text { Showed a significant decrease in elevated NO } \\
\text { level in streptozotocin induced pancreatic } \beta \\
\text { cells at } 100 \mathrm{mg} / \mathrm{kg} . \text {. }\end{array}$ & $\begin{array}{l}\text { Adewole and } \\
\text { Caxton- } \\
\text { Martins, } \\
2006\end{array}$ \\
\hline $\begin{array}{l}\text { Annona purpurea Moc. and } \\
\text { Sessé ex Dunal }\end{array}$ & Leaves & $\begin{array}{l}\text { 7-hydroxy-dehydrothalicsimidine, } \\
\text { thalicsimidine, } \\
\text { N-methyllaurotetanine, lirinidine, } \\
\text { N-methylasimilobine }\end{array}$ & Alkaloids & $\begin{array}{l}\text { PAF-induced platelet aggregation was inhibited } \\
\text { at a concentration range of } 20-50 \mu \mathrm{M}\end{array}$ & $\begin{array}{l}\text { Chang et al., } \\
1998\end{array}$ \\
\hline \multirow[t]{2}{*}{ Annona reticulata $\mathrm{L}$} & Bark & Kaur-16-en-19-oic acid & Ent-kaurane Diterpenoid & $\begin{array}{l}\text { Hot plate reaction time was increased, } \\
\text { reduction in acetic acid-induced abdominal } \\
\text { writhing and carrageenan induced rat paw } \\
\text { edema was observed at } 20 \mathrm{mg} / \mathrm{kg}\end{array}$ & $\begin{array}{l}\text { Chavan et al., } \\
2012\end{array}$ \\
\hline & Bark & Methanolic extract & - & 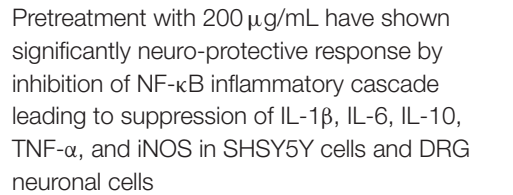 & $\begin{array}{l}\text { Kandimalla } \\
\text { et al., } 2017\end{array}$ \\
\hline
\end{tabular}


TABLE 2 | Continued

\begin{tabular}{|c|c|c|c|c|c|}
\hline Plant name & Part used & Isolate compound/Extract & Class & Mechanism of action/Conclusion & References \\
\hline & Leaves & $\begin{array}{l}\text { Kaurenoic acid, taraxerol, } 16 \alpha \text { - } \\
\text { hydro-19-al-ent-kauran-17-oic } \\
\text { acid, } \\
6 \beta \text {-hydroxystigmast-4-en-3-one, } \\
\text { and } 17 \text {-acetoxy-16 } \beta \text {-ent-kauran- } \\
\text { 19-oic acid } 24 \text {, } \\
16 \alpha \text {-hydro-ent-kauran-17,19- } \\
\text { dioic } \\
\text { acid }\end{array}$ & ent-kaurane diterpenoids & $\begin{array}{l}\text { Significant } \mathrm{NO} \text { and superoxide anion } \\
\text { generation inhibitory activity was observed at } \\
\mathrm{IC}_{50} \text { value ranging from } 5.25 \text { to } 8.65 \mu \mathrm{M}\end{array}$ & $\begin{array}{l}\text { Thang et al., } \\
2013 b\end{array}$ \\
\hline Annona senegalensis Pers & Leaves & Ethanolic extract & - & $\begin{array}{l}\text { Deceased the production of neutrophils, } \\
\text { eosinophil and macrophages at } 7 \mathrm{mg} / \mathrm{kg}\end{array}$ & $\begin{array}{l}\text { Yeo et al., } \\
2011\end{array}$ \\
\hline \multirow[t]{5}{*}{ Annona squamosa $L$} & Bark & Caryophyllene oxide & Sesquiterpentine & $\begin{array}{l}\text { Late phase of paw licking edema was } \\
\text { significantly reduced at the dose of } 50 \mathrm{mg} / \mathrm{kg}\end{array}$ & $\begin{array}{l}\text { Chavan et al., } \\
2010\end{array}$ \\
\hline & Seeds & $\begin{array}{l}\text { Cyclosquamosin and } \\
\text { met-cherimolacyclopeptide }\end{array}$ & Cyclopeptides & $\begin{array}{l}\text { IL- } 6 \text { and TNF- } \alpha \text { production was suppressed in } \\
\mathrm{J} 774 \mathrm{~A} \text { with an } \mathrm{IC}_{50} \text { value of } 1.22 \text { and } 9.2 \mu \mathrm{M}\end{array}$ & $\begin{array}{l}\text { Dellai et al., } \\
2010\end{array}$ \\
\hline & Fruit & $\begin{array}{l}\text { Fanlizhicyclopeptide a } \\
\text { fanlizhicyclopeptide }\end{array}$ & Cyclopeptides & $\begin{array}{l}\text { Pro-inflammatory cytokine production was } \\
\text { inhibited by } 32 \text { and } 27 \% \text {, TNF- } \alpha \text { by } 51 \text { and } 57 \\
\% \text {, and IL- } 6 \text { by } 66 \text { and } 49 \% \text { at } 25 \mu \mathrm{M}\end{array}$ & $\begin{array}{l}\text { Wu P. et al., } \\
2014\end{array}$ \\
\hline & Stem & $\begin{array}{l}\text { 16beta, } 17 \text {-dihydroxy-ent- } \\
\text { kauran-19-oic } \\
\text { acid }\end{array}$ & Ent-kaurane & $\begin{array}{l}\text { Suppressed the degranulation of neutrophils } \\
\text { were suppressed through immobilization of } \\
\text { cytosolic calcium in a concentration dependent } \\
\text { manner at } \mathrm{IC}_{50} \text { value of } 12.52 \mu \mathrm{M}\end{array}$ & $\begin{array}{l}\text { Yeh et al., } \\
2005\end{array}$ \\
\hline & Leaves & - & - & $\begin{array}{l}\text { Significant inhibition of NO }(73.64 \%) \text {, moderate } \\
\text { reduction in superoxide ( } 89.77 \%) \text {, and lipid } \\
\text { peroxidation ( } 99.02 \%) \text { was observed due to its } \\
\text { potent scavenging activity at } 1,000 \mu \mathrm{g} / \mathrm{ml}\end{array}$ & $\begin{array}{l}\text { Shirwaikar } \\
\text { et al., } 2004\end{array}$ \\
\hline Annona sylvatica A.St.-Hil & Leaves & $\begin{array}{l}\text { Hinesol, z-caryophyllene, } \\
\text { beta-maaliene, }\end{array}$ & Sesquiterpenes & $\begin{array}{l}\text { Leukocytes migration was inhibited at a } \\
\text { concentration range of } 36.04-45.37 \mu \mathrm{g} / \mathrm{mL}\end{array}$ & $\begin{array}{l}\text { Formagio } \\
\text { et al., } \\
2013 a, b c\end{array}$ \\
\hline Annona vepretorum & Leaves & crude ethanolic extract & - & $\begin{array}{l}\text { Oral dose of } 25,50,100 \mathrm{mg} / \mathrm{kg} \text { inhibited the } \\
\text { release of inflammatory mediators and } \\
\text { leukocyte migration is inhibited by } 59,65 \text {, and } \\
79 \% \text {, respectively }\end{array}$ & $\begin{array}{l}\text { Silva et al., } \\
2015\end{array}$ \\
\hline $\begin{array}{l}\text { Cyathostemma Argenteum } \\
\text { (Blume) J.Sinclair }\end{array}$ & & $\begin{array}{l}4^{\prime}, 6^{\prime} \text {-dihydroxy-2', } 4 \text { - } \\
\text { dimethoxy-5'-(2"'-hydroxybenzyl) } \\
\text { and dihydrochalcone } \\
\text { dihydrochalcone, } 4^{\prime}, \\
6^{\prime} \text {-dihydroxy-2', } 4 \text { - } \\
\text { dimethoxydihydrochalcone }\end{array}$ & Chalcone & $\begin{array}{l}\text { Release of inflammatory mediators and } \\
\text { leukocyte migration was significantly inhibited } \\
\text { in rats at concentration of } 1 \mathrm{mg} / \mathrm{ear}\end{array}$ & $\begin{array}{l}\text { Somsrisa } \\
\text { et al., } 2013\end{array}$ \\
\hline Duguetia chrysocarpa Mass & Fruit & Discretamine & Alkaloid & $\begin{array}{l}\text { Acetic acid-induced writhing, formalin and hot } \\
\text { plate tests has shown significant results }\end{array}$ & $\begin{array}{l}\text { Almeida et al., } \\
2012\end{array}$ \\
\hline $\begin{array}{l}\text { Enicosanthellum pulchrum } \\
\text { (King) Heusden }\end{array}$ & Roots & Ethyle acetate extract & - & $\begin{array}{l}\text { Produces platelet activating factor antagonistic } \\
\text { activity at } 85.6 \% \text { inhibition at } 250 \mathrm{mg} / \mathrm{kg} \text { oral } \\
\text { dose }\end{array}$ & $\begin{array}{l}\text { Nordin et al., } \\
2012\end{array}$ \\
\hline $\begin{array}{l}\text { Fissistigma cavaleriei } \\
\text { (H.Lév.) Rehder }\end{array}$ & Root & $\begin{array}{l}\text { Compound } 1 \text { (name not } \\
\text { illustrated by author) }\end{array}$ & Alkaloid & $\begin{array}{l}\text { Suppress COX2 expression at } I_{50} \text { value of } \\
32 \mu \mathrm{g} / \mathrm{mL}\end{array}$ & $\begin{array}{l}\text { Yang Z. et al., } \\
2012\end{array}$ \\
\hline \multirow[t]{3}{*}{$\begin{array}{l}\text { Fissistigma Oldhamii } \\
\text { (Hemsl.) Merr }\end{array}$} & Stem & Crude ethanolic extracts & - & $\begin{array}{l}\text { TNF- } \alpha \text { and IL- } 6 \text { production and released } \\
\text { suppressed at } 50 \mu \mathrm{g} / \mathrm{mL}\end{array}$ & $\begin{array}{l}\text { Ge et al., } \\
2013\end{array}$ \\
\hline & Stem & Isopedicin & Flavonoid & $\begin{array}{l}\text { The production of ROS in neutrophils were } \\
\text { inhibited due to the elevation of cellular cAMP } \\
\text { and activation of protein kinase A through its } \\
\text { inhibition of CAMP-specific phosphodiesterase } \\
\text { at an } \mathrm{IC}_{50} \text { value of } 0.34 \mu \mathrm{M}\end{array}$ & $\begin{array}{l}\text { Hwang et al., } \\
2009\end{array}$ \\
\hline & Leaves & $\begin{array}{l}7^{\prime}-\left(3^{\prime}, 4^{\prime} \text {-dihydroxyphenyl)-n-[(4- }\right. \\
\text { methoxyphenyl) ethyl] } \\
\text { propenamide }(z 23)\end{array}$ & - & $\begin{array}{l}\text { Z23 has decreased the gene expression of } \\
\text { COX2 and iNOS at a concentration range of } \\
6.25-20 \mu \mathrm{M}\end{array}$ & $\begin{array}{l}\text { Hu et al., } \\
2008\end{array}$ \\
\hline
\end{tabular}


TABLE 2 | Continued

\begin{tabular}{|c|c|c|c|c|c|}
\hline Plant name & Part used & Isolate compound/Extract & Class & Mechanism of action/Conclusion & References \\
\hline $\begin{array}{l}\text { Goniothalamus. clemensii, } \\
\text { Ban Goniothalamus. Woodii } \\
\text { Mex ex Mat- Salleh, }\end{array}$ & Bark & Bark oil & $\begin{array}{l}\text { Sesquiterpene and } \\
\text { Sesquiterpenoid }\end{array}$ & $\begin{array}{l}\text { PAF and arachidonic acid activity was } \\
\text { significantly inhibited at } 20 \mu \mathrm{g} / \mathrm{mL}\end{array}$ & $\begin{array}{l}\text { Vendramini- } \\
\text { Costa et al., } \\
2014\end{array}$ \\
\hline
\end{tabular}

Goniothalamus. velutinus

Mex ex Mat- Salleh and

Goniothalamus. tapis Miq

Bark and Goniothalamin Styryl-lactones
root

Bark Goniothalamin

(+)-Goniothalamin and

(+)-isoaltholactone

King and Goniothalamus

tapis Miq

Goniothalamus. clemensii, Bark and

Ban Goniothalamus. Woodii Root

Mex ex Mat- Salleh,

Goniothalamus, velutinus

Mex ex Mat- Salleh and

Goniothalamus. tapis Miq

Guatteria australis A.St.-Hil

Goniothalamus

macrophyllus Bloom Hook.f. bark

and Thomas

Meiocarpidium Lepidotum Bark

Lepidotum (Oliv) Engl. and

Diels

Melodorum fruticosum Lour Leaves

Mitrella kentia (Blume) Miq Leaves

Leaves

Miliusa balansae Finet and Leaves

Gapnep

Monodora myristica

(Geartn.)Dunal

Monodora tenuifolia Benth Seed

Seed

Oxandra xylopioides Diels

Leaves

Bark and 3-oxo-berenjenol)
Methanolic crude extract

Berenbjenol

Berenjenol (berenjenol acetate

Acetylmelodorinol, chrysin and polycarpol, benzoquinone and stigmasterol

Desmosdumotin

Milbasides A, B and C

Hydro-ethanolic Extract

Cycloartane triterpene

Cycloartane triterpene

Alkaloid

Chalcone

$-$

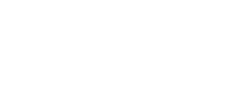

Styryl-lactones and sesquiterpene lactone

Essential oils

Essential oils

Styryl-lactone

Triterpines

Phenolic amide

MegastigmaneGlycosides

exhibited maximum effect against lipid $100 \mu \mathrm{M}$ peroxidation and free radical generation, exhibited significant antioxidant activity in NO induced lipid peroxidation

Significantly reduced the IL-1 production by 72 Rojano et al. and $81 \%$ and carrageenan induced paw 2007 edema by 64 nd 43 \& at concentration of

Expression of COX-2 and iNOS was reduced Aquila et al., by 65 and $80 \%$ at $50 \mu \mathrm{M}$

Vendramini-

Costa et al. 2014 induction property at concentration range of $25 \mu \mathrm{g} / \mathrm{mL}$ and TNF- $\alpha$ was down regulated and Costa et al. $10-50 \mu \mathrm{g} / \mathrm{mL}$

Significant PAF receptor antagonist activity was Moharam $19.7,46.5 \mu \mathrm{M}$

PAF receptor antagonist activity and PAF Moharam induced platelet aggregation was significantly et al., 2010a inhibited with an $\mathrm{IC}_{50}$ value of 93.3 and $87.7 \mu \mathrm{g} / \mathrm{ml}$

Slight anti-oxidant activity was observed at Siqueira et al., $250 \mu \mathrm{g} / \mathrm{ml}$.

2015

GTN inhibited TNF $\alpha$ induced NF-кb activation Orlikova et al., with an $\mathrm{IC}_{50}$ value of $5 \mu \mathrm{M}$

Significantly reduced the writhing, Meddah carrageenan-induced hyperalgesia in mice at et al., 2013 inhibition of neutrophils at an $\mathrm{IC}_{50}$ value 2013

Significant and concentration dependent Saadawi inhibition of PAF, PGE 2 and thromboxane B2 at et al., 2012

Selectively inhibited COX-2 by $29.5 \%$ and Sidahmed $34.8 \%$ at 250 and $500 \mathrm{ng} / \mathrm{ml} \quad$ et al., 2013 Inhibition of carrageenan-induced paw edema, Ishola et al., and xylene-induced ear edema was 2016 significantly reduced at a concentration range of 50-200 mg/kg

Njoku, 2007 2009 
TABLE 2 | Continued

\begin{tabular}{|c|c|c|c|c|c|}
\hline Plant name & Part used & Isolate compound/Extract & Class & Mechanism of action/Conclusion & References \\
\hline \multirow[t]{5}{*}{$\begin{array}{l}\text { Polyalthia longifolia (Sonn.) } \\
\text { Thwaites }\end{array}$} & Bark & $\begin{array}{l}\text { 16-hydroxycleroda-13-ene- } \\
\text { 15,16-olide-3-one }\end{array}$ & Clerodane diterpenoid & $\begin{array}{l}\text { Superoxide Anion generation was inhibited with } \\
\text { an } \mathrm{IC}_{50} \text { value of } 0.60 \pm 0.09 \mu \mathrm{g} / \mathrm{ml}\end{array}$ & $\begin{array}{l}\text { Chang et al., } \\
2006\end{array}$ \\
\hline & Leaves & $\begin{array}{l}\text { PI3s [6-hydroxycleroda- } \\
\text { 3,13(14)e-dien- 15-oic } \\
\text { acid] }\end{array}$ & Clerodane diterpenoid & $\begin{array}{l}\text { Neutrophil respiratory burst and superoxide } \\
\text { anion generation was significantly inhibited at } \\
3.06 \pm 0.20 \text { and } 3.30 \pm 0.48 \mu \mathrm{M} \text {, respectively }\end{array}$ & $\begin{array}{l}\text { Chang et al., } \\
\text { 2008; Tanna } \\
\text { et al., } 2009\end{array}$ \\
\hline & Unripe fruit & $\begin{array}{l}\text { 16-hydroxycleroda-3,13-dien- } \\
\text { 15,16-olide (6) and } \\
\text { 16-oxocleroda-3,13-dien-15-oic } \\
\text { acid (7) }\end{array}$ & Diterpenes & $\begin{array}{l}\text { At } 10 \mu \mathrm{M} \text { iNOS production was significantly } \\
\text { inhibited by } 81.1 \text { and } 86.3 \% \text { with an } \mathrm{IC}_{50} \text { value } \\
\text { of } 1 \mu \mathrm{M}\end{array}$ & $\begin{array}{l}\text { Wu T. H. } \\
\text { et al., } 2014\end{array}$ \\
\hline & Leaf & Ethanolic extract & - & $\begin{array}{l}\text { Maximum NO scavenging activity was } 70.67 \% \\
\text { with an } \mathrm{IC}_{50} \text { value of } 167 \mu \mathrm{g} / \mathrm{ml}\end{array}$ & $\begin{array}{l}\text { Saha et al., } \\
2008\end{array}$ \\
\hline & Leaves & $\begin{array}{l}\text { 6-hydroxycleroda-3,13-dien- } \\
\text { 15,16-olide } \\
\text { (pl3) }\end{array}$ & Diterpenes & $\begin{array}{l}\text { Pre-treatment with } 10 \mu \mathrm{g} / \mathrm{ml} \text { pl3 notably } \\
\text { decreased the production of } \mathrm{NO}, \mathrm{PGE}_{2} \text {, iROS, } \\
\text { and TNF } \alpha \text {. Moreover, gene expression of } \\
\mathrm{NF}-\kappa \mathrm{B} \text { p } 65 \text {, COX-2, and iNOS was also } \\
\text { suppressed }\end{array}$ & $\begin{array}{l}\text { Shih et al., } \\
2010\end{array}$ \\
\hline Polyalthia parviflora Ridl. & Leaves & $\begin{array}{l}13 \text { 6s-styryllactones, } \\
\text { 6s-styrylpyrones } \\
\text { and1s-phenylpyranopyrones }\end{array}$ & Styryllactones & $\begin{array}{l}\text { Superoxide anion generation and elastase } \\
\text { release from human neutrophils was } \\
\text { suppressed were inhibited with an } I_{50} \text { value } \\
\text { of } 30.1 \pm 2.5 \text { and } 21.2 \pm 2.2 \mu \mathrm{M} \text {, respectively }\end{array}$ & $\begin{array}{l}\text { Liou et al., } \\
2014\end{array}$ \\
\hline $\begin{array}{l}\text { Pseuduvaria macrophylla } \\
\text { (Oliv.) Merr }\end{array}$ & Bark & Crude methanolic extract & - & $\begin{array}{l}\text { Diabetic rats treated with } 400 \mathrm{mg} / \mathrm{kg} \\
\text { significantly inhibited the production of } \\
\text { pro-inflammatory cytokines including TNF- } \alpha \text {, } \\
\text { IL-1 } 1 \beta \text {, IL- } 6\end{array}$ & $\begin{array}{l}\text { Arya et al., } \\
2014\end{array}$ \\
\hline $\begin{array}{l}\text { Pseuduvaria monticola } \\
\text { J.Sinclair }\end{array}$ & Bark & Crude ethanolic extract & - & $\begin{array}{l}\text { Daily administration of } 500 \mathrm{mg} / \mathrm{kg} \text { for } 45 \text { days } \\
\text { down regulated the levels of oxidative stress } \\
\text { and pro-inflammatory cytokines by inhibiting } \\
\text { the translocation of NF-kB in type } 2 \text { diabetic rat } \\
\text { model }\end{array}$ & $\begin{array}{l}\text { Taha et al., } \\
2014\end{array}$ \\
\hline $\begin{array}{l}\text { Polyalthia cerasoides } \\
\text { (Roxb.) Bedd }\end{array}$ & Leave & Methenolic leave extract & - & $\begin{array}{l}\text { Hydroxyl radical, superoxide anion scavenging, } \\
\text { and potent reducing activity was observed in } \\
\text { rats treated with } 40 \mathrm{mg} / \mathrm{kg} \text { of extract }\end{array}$ & $\begin{array}{l}\text { Ravikumar } \\
\text { et al., } 2008\end{array}$ \\
\hline \multirow[t]{2}{*}{ Rollinia mucosa (Jacq) Baill } & $\begin{array}{l}\text { Leave and } \\
\text { seeds }\end{array}$ & $\begin{array}{l}\text { Magnolin, epiyangambin, } \\
\text { yangambin }\end{array}$ & Lignans & $\begin{array}{l}\text { Significant PAF receptor antagonist activity was } \\
\text { recorded at a } \mathrm{IC}_{50} \text { range of } 1.1-6.7 \mu \mathrm{M}\end{array}$ & $\begin{array}{l}\text { Faria Lua } \\
\text { Figueiredo } \\
\text { et al., } 1999\end{array}$ \\
\hline & Stems & Romucosine A and D & Alkaloid & $\begin{array}{l}\text { At } 100 \mu \mathrm{g} / \mathrm{ml} \text { maximum PAF receptor } \\
\text { antagonist activity was recorded }\end{array}$ & $\begin{array}{l}\text { Kuo et al., } \\
2001\end{array}$ \\
\hline Toussaintia orientalis Verdc & $\begin{array}{l}\text { Stem, root } \\
\text { and bark }\end{array}$ & Aristolactam aii, aristolactam bii & $\begin{array}{l}\text { Aristolactam alkaloid } \\
\text { toussa lactam }\end{array}$ & $\begin{array}{l}\text { Maximum inhibition of histamine release from } \\
\text { mast cells via stabilizing the cell membrane } \\
\text { was observed at } \mathrm{IC}_{50} \text { value of } 5.1 \text { and } 11.9 \mu \mathrm{M}\end{array}$ & $\begin{array}{l}\text { Odalo et al., } \\
2010\end{array}$ \\
\hline Uvaria chamae P.Beauv & $\begin{array}{l}\text { Whole } \\
\text { plant }\end{array}$ & Methanolic crude extract & - & $\begin{array}{l}\text { Pretreatment with } 400 \mathrm{mg} / \mathrm{kg} \text { for } 6 \mathrm{~h} \text { inhibited } \\
\text { paw circumference in the carrageenan- and } \\
\text { formaldehyde-induced in rat paw oedema tests }\end{array}$ & $\begin{array}{l}\text { Popoola } \\
\text { et al., } 2016\end{array}$ \\
\hline Uvaria flexuosa Ast \& Jovet & Leaves & Flexuvarol b and chrysin & Flavones & $\begin{array}{l}\text { Superoxide anion generation and elastase } \\
\text { release from human neutrophils was } \\
\text { suppressed at an } \mathrm{IC}_{50} \text { value of } 2.25-5.55 \mu \mathrm{M}\end{array}$ & $\begin{array}{l}\text { Hsu et al., } \\
2016\end{array}$ \\
\hline $\begin{array}{l}\text { Uvaria grandiflora Roxb. Ex } \\
\text { Hornem }\end{array}$ & Stem & $(-)$-Zeylenol & $\begin{array}{l}\text { Polyoxygenated } \\
\text { cyclohexene }\end{array}$ & $\begin{array}{l}\text { Pretreatment with } 1 \mathrm{mg} / \mathrm{ear} \text { deceased the } \\
\text { xylene induced ear edema in time dependent } \\
\text { manner. }\end{array}$ & $\begin{array}{l}\text { Seangphakdee } \\
\text { et al., } 2013\end{array}$ \\
\hline $\begin{array}{l}\text { Xylopiadiscreta (L.f) } \\
\text { Sprague \& hutch }\end{array}$ & $\begin{array}{l}\text { Leave and } \\
\text { seed }\end{array}$ & Crude leaf methanol extract & - & $\begin{array}{l}\mathrm{IL}-12, \mathrm{TNF} \alpha \text {, and IL-10 production was } \\
\text { deceased in leishmania infected macrophages } \\
\text { with a Sensitivity index of } 64.8 \mathrm{~J} 774 \text { cells. }\end{array}$ & $\begin{array}{l}\text { López et al., } \\
2009\end{array}$ \\
\hline $\begin{array}{l}\text { Xylopia aethiopica (Dunal } \\
\text { A.Rich) }\end{array}$ & Fruit & $\begin{array}{l}\text { Ethanolic extract and xylopic } \\
\text { acid }\end{array}$ & - & $\begin{array}{l}\text { Pretreatment with } 300 \text { mg/kg significantly } \\
\text { reduced by } 49.84 \pm 3.94 \text { and } 43.62 \pm 1.01 \% \text {, } \\
\text { respectively }\end{array}$ & $\begin{array}{l}\text { Woode et al., } \\
2012 \mathrm{~b} \text {; Obiri } \\
\text { and Osafo, } \\
2013\end{array}$ \\
\hline
\end{tabular}


TABLE 2 | Continued

\begin{tabular}{|c|c|c|c|c|c|}
\hline Plant name & Part used & Isolate compound/Extract & Class & Mechanism of action/Conclusion & References \\
\hline & Dried Fruit & Water extract & - & Potent antioxidant activity & $\begin{array}{l}\text { Odukoya } \\
\text { et al., } 2005\end{array}$ \\
\hline $\begin{array}{l}\text { Xylopia laevigata (Mart.) } \\
\text { R.E.Fr. }\end{array}$ & Leaf & Hydrodistilled oil & $\begin{array}{l}\text { Leaf containing essential } \\
\text { oils }\end{array}$ & $\begin{array}{l}\text { Pre-treatment with } 50 \mathrm{mg} / \mathrm{kg} \text { of extract } \\
\text { significant reduced carrageenan-induced } \\
\text { peritonitis and carrageenan induced hindpaw } \\
\text { edema in mice }\end{array}$ & $\begin{array}{l}\text { Queiroz et al., } \\
2014\end{array}$ \\
\hline $\begin{array}{l}\text { Xylopia langsdorffiana St } \\
\text { Hilaire and Tulasne }\end{array}$ & Leaves & Ethanolic and hexane extract & - & $\begin{array}{l}\text { Pre-treatment with } 50 \mathrm{mg} / \mathrm{kg} \text { produced } \\
\text { gastroprotective effect by inhibiting the } \\
\text { production of NO from } 85 \text { to } 24 \%\end{array}$ & $\begin{array}{l}\text { de } \\
\text { Albuquerque } \\
\text { Montenegro } \\
\text { et al., } 2014\end{array}$ \\
\hline \multirow[t]{2}{*}{ Xylopia parviflora Spruc } & Seeds & $\begin{array}{l}\text { Water, ethanolic, and } \\
\text { hydroethanolic extracts }\end{array}$ & - & $\begin{array}{l}\text { Highest inhibition of LDL oxidation and NO } \\
\text { scavenging activity was observed at a } \\
\text { concentration range of } 250-500 \mu \mathrm{g} / \mathrm{ml}\end{array}$ & $\begin{array}{l}\text { Kuate et al., } \\
2011\end{array}$ \\
\hline & Fruits & Hydrodistilled oil & Essential oil & $\begin{array}{l}\text { A dose-dependent decrease in } \mathrm{NO} \text { production } \\
\text { with an } \mathrm{IC}_{50} \text { of } 7.47 \mu \mathrm{g} / \mathrm{ml}\end{array}$ & $\begin{array}{l}\text { Woguem } \\
\text { et al., } 2014\end{array}$ \\
\hline
\end{tabular}

that inhibiting the $\mathrm{PGE}_{2}$ production through COX enzyme is responsible for the anti-inflammatory activity of Asprin (Smith and Willis, 1971; Moncada et al., 1976). Later the discovery of two different isoforms of COX enzyme COX 1 and COX 2 further highlighted the role of PGs in inflammation. COX-1 is constitutive, expressed on platelets and gastric partial cells, responsible for modulating platelet aggregation and maintaining the gastric mucosal protective lining. Whereas, COX-2 is an inducible enzyme with cellspecific distribution. COX-2 activation give rise to prostaglandins responsible for producing classical sign and symptoms of inflammation including, hyperalgesia and swelling (Harrington et al., 2008; Rouzer and Marnett, 2009). Numerous internal and external factors including stress growth factors, mitogens, and inflammatory cytokines may cause the up regulations of COX-2 gene expression. This up regulation may give rise to development and progression of chronic inflammation, angiogenesis, and cancer metastasis (Williams et al., 1999; Dannenberg et al., 2001). Henceforth, natural products with COX-2 inhibitory activity and downregulation of over expressed COX-2 expression can be a noteworthy pharmacological therapeutic options for treatment inflammatory conditions.

Annonaceous acetogenins are usually 35-77 carbon compounds, produced by polyketide pathway (Liaw et al., 2010). First acetogenins was discovered in 1982 and ever since series of investigation has been carried on this fascinating class of natural product due to its unique structure and versatile bio-activities (Chang and Wu, 2001; Kojima and Tanaka, 2009; de Sousa et al., 2010; Chen et al., 2012; Zhang et al., 2015). In the quest to explore phytochemicals with COX 2 inhibitory activity, isodesacetyluvaricin (5) (Figure 1), an annonaceous acetogenin from Annona glabra was evaluated on A431 carcinoma cells (Wu et al., 2012). The addition of $25 \mu \mathrm{g} / \mathrm{mL}$ of Epidermal Growth Factor (EGF) resulted in increased expression of COX-2 mRNA, without effecting COX-1. Upon addition of isodesacetyluvaricin (5), expression of COX-2 mRNA was significantly reduced in a dose dependent manner, without affecting COX 1 expression. Upon further investigation, it was proposed that selective COX-2 inhibition was due to the suppression of promotor activity of element binding factor (CREB) and the nuclear factor of activated $\mathrm{T}$ cells (NFAT) responsible for the EGF-mediated transcriptional activation of COX-2 (Duque et al., 2005; Yiu and Toker, 2006). Isodesacetyluvaricin(5) significantly inhibited $(P<0.05)$ CREB and NFAT at a concentration ranging $1-$ $5 \mu \mathrm{M}$ further cementing the proposed mechanism. However, an alternative mechanism was also proposed, involving the phosphorylation of CREB by protein kinase A- dependent Rap1-extracellular-signal-related kinase and dephosphorylation of NFAT (Iñiguez et al., 2000; Chun and Surh, 2004). Alkaloid (compound 1; name not specified by author) isolated from Fissistigma cavaleriei root showed corresponding activity (Yang Z. et al., 2012). Colorimetric screening assay revealed that compound 1 has significant angiogenic property due to its selective COX-2 inhibition. Subsequently, Hu et al. (2008) further supported the COX-2 inhibitory activity of Fissistigma oldhamii. $\quad 7^{\prime}$-(3', 4'-Dihydroxyphenyl)-n-[(4-methoxyphenyl) ethyl] propenamide (Z23) (6) (Figure 1) isolated from the leaves of $F$. oldhamii reduced the COX 2 gene expression in LPS induced Raw 256.7 macrophage cells. Moreover, Aquila et al. (2009) reported that cycloartane triterpene, berenjenol (7) (Figure 1) isolated from Oxandra xylopioides, significantly $(p<0.05)$ decreased the COX-2 gene expression at $50 \mu \mathrm{M}$.

Mitrella kentia is a tree-climbing liana from Annonaceae family. It is proposed that it possesses significant antiinflammatory activity due to presence various bioactive compounds including, isoquinoline alkaloids, chalcones, and essential oils. Saadawi et al. (2012) evaluated the PGE 2 inhibitory activity of $M$. kentii using highly sensitive radioimmunoassay technique. Among all the isolated compounds chrysin (8) (Figure 1) showed dose dependent $\mathrm{PGE}_{2}$ inhibition with an $\mathrm{IC}_{50}$ value of $25.5 \mu \mathrm{M}$. Results from another report further supported the corresponding $M$. kenti activity against $\mathrm{PGE}_{2}$. Desmosdumotin C (9) (Figure 1) a new isolated bioactive compound from $M$. kentii revealed gastro-protective activity by decreasing gastric ulcers area, edema, and leukocyte infiltration, 

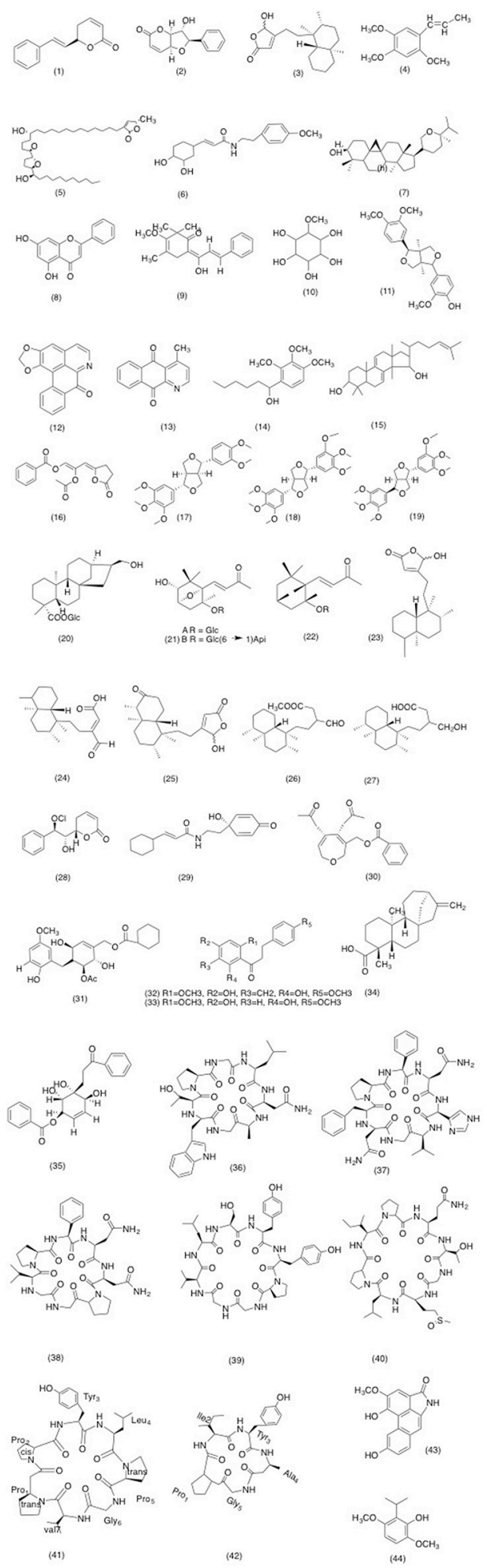

FIGURE 1 | Structure of compounds isolated from Annonaceae species with potent anti-inflammatory activity (1) Goniothalamin, (2) Altholactone, (3) 6-hydroxycleroda-3,13-dien-15,16-olide (PL3), (4) alpha asarone, (5) Isodesacetyluvaricin, (6) 7' -(3',4'-dihydroxyphenyl)-n-[(4-methoxyphenyl) ethyl] Propenamide (z23), (7) berenjenol, (8) chrysin (9) desmosdurnotin C, (10)

(Continued)
FIGURE 1 | Continued

Quebrachitol, (11) Phylligenin, (12) liriodenine, (13) Cliestopholine, (14) dehydroanonaine 1-(2', 3', 4'-Trimethoxyphenyl)hexan-1-ol, (15) Polycarpol, (16) acetylmelodorinol, (17) magnolin, (18) epiyangambin, (19) yangambi, (20) 7 beta, 17-dihydroxy-ent-kaur-15-en-19-oic acid 19-O-beta-D-glucopyranoside ester, (21) Milbasides A and B, (22) Milbasides C, (23)

16-hydroxycleroda-3,13-dien-15,16-olid, (24) 16-oxocleroda-3,13-dien-15-oic acid, (25) 16-hydroxycleroda-13-ene-15,16-olide-3-one, (26)

16-oxocleroda-3,13(14)E-dien-15-oic acid methyl ester, (27)

16-hydroxycleroda-3,13(14)E-dien-15-oic acid, (28) (28) Parvistones, (29) melodamide A, (30) 3-methyl-4,5-dihydro-oxepine, (31) flexuvarol B, (32) $4^{\prime} 6^{\prime}$-dihydroxy-2',4-dimethoxy-5'-(2"-hydroxybenzyl) dihydrochalcone, (33) dihydrochalcone, 4', 6'-dihydroxy-2', 4-dimethoxydihydrochalcone, (34) Kaur-16-en-19-oic acid, (35) Zeylenol, (36) Cyclomontanins A (37)

Cyclomontanins B (38) Cyclomontanins (39) Cyclomontanins D (40)

cherimolacycopeptide B, (41) fanlizhicyclopeptide B, (43) Aristololactam.

which could be attributed intervention with anti $\mathrm{H}$ pylori and COX-2 inhibitory pathway (Sidahmed et al., 2013).

\section{Platlet-Activation Factor (PAF) Inhibition}

PAF plays a significant role in carrying out several physiological functions. Unfortunately when body faces mechanical stress, trauma or exposed to toxin the concentrations of PAF rises significantly. These elevated levels may give rise to numerous pathophysiological conditions such as inflammation (Stafforini et al., 2003), allergy (Petersen et al., 1997), asthma (Kasperska-Zajac et al., 2008), and thrombosis (Zhang et al., 2001). Lately, it is proposed that in order to carry out its pathophysiological functions it is imperative for PAF to specifically binds to its receptors (Esquenazi and Bazan, 2010). Therefore, compounds with PAF receptor antagonistic activity can be used as a good therapeutic option to treat PAF associated inflammatory condition (Moharam et al., 2010b). Moreover, PAF and prostanoins share common pathway hence $\mathrm{TXA}_{2}$ may acts as a PAF mediator and produce the symptoms associated with PAF elevations (Badr et al., 1989). In addition, increased $\mathrm{PGE}_{2}$ synthesis was observed in PAF treated rat meningeal cells suggesting its role in the prostanoids secondary production (Arribas-Gómez et al., 1995).

A few species of Annonaceae have displayed compelling PAF receptor antagonistic activity. For instance, Jantan et al. (2005) evaluated PAF inhibitory activity of 49 methanolic extracts from 37 Malaysian indigenous plant species. Their inhibitory effects were evaluated using ${ }^{3} \mathrm{H}-\mathrm{PAF}$ as a ligand. 6 Zingiberaceae species, two Cinnamomum species and one Annonaceae species (Goniothalamus malayanus) were reported as novel PAF antagonists, as they exhibited noteworthy inhibitory effects with $\mathrm{IC}_{50}$ values ranging from 1.2 to $18.4 \mu \mathrm{g} / \mathrm{mL}$. Furthermore, two alkaloids and aporphine alkaloids isolated from the twigs of Mitrephora vulpine were evaluated using ${ }^{3} \mathrm{H}-\mathrm{PAF}$ ligand model (Moharam et al., 2010b). Out of all the isolated compounds phylligenin (10) and quebrachitol (11) (Figure 1) managed to antagonize PAF receptor in concentration depended manner with $\mathrm{IC}_{50}$ values of 13.1 and $42.2 \mu \mathrm{M}$, respectively. Whereas, the phylligen have an $\mathrm{IC}_{50}$ value comparable to cedrol $(10.2 \mu \mathrm{M})$, a potent PAF 
antagonist. In continuation to previous work Moharam et al. (2010a) focused on the essential oils isolated from five Goniothalamus species (G. tapisoides, G. velutinus, G. clemensii, G. tapis, and G. woodii). Essential oil from the bark of $G$. clemensii, G. woodii, G. velutinus, and the root oil of G. tapis were able to show significant antagonist activity with $\mathrm{IC}_{50}$ values ranging from 3.5 to $10.5 \mu \mathrm{g} / \mathrm{ml}$. It was proposed that the presence of sesquiterpenes and sesquiterpenoids were responsible for producing potent PAF receptor antagonistic activity. Furthermore, Nordin et al. (2012) highlighted the similar bioactivity of E. pulchrum first time ever. Among the extracts examined, ethyl acetate extract has shown superior antagonist activity with $85.6 \%$ inhibition. Moreover, liriodenine (12), cleistopholine (13) and dehydroanonaine 1-(2', $3^{\prime}$, $4^{\prime}$-trimethoxyphenyl)hexan-1-ol(14) (Figure 1) isolated compounds from the same fraction were the strongest to antagonize PAF receptor with an $\mathrm{IC}_{50}$ values of 26.6, 50.2, and $45.4 \mu \mathrm{M}$, respectively. Saadawi et al. (2012) reported that polycarpol (15) and acetylmelodorinol (16) (Figure 1) isolated from Mitrella kentii (Bl.) have shown similar dose dependent inhibitory effects with $\mathrm{IC}_{50}$ values of 24.3 and $24.5 \mu \mathrm{M}$, respectively. Faria Lua Figueiredo et al. (1999) reported parallel activity for magnolin (17), epiyangambin (18), yangambin (19) (Figure 1) and furofuranic lignans isolated from leaves of Rollinia deliciosa.

\section{Inducible Nitrous Oxide (iNOS) Inhibition}

Numerous vascular diseases are often supplemented with inflammation, which may affect the production of peroxynitrite and protein nitration and may lead to irreversible DNA damage and apoptosis (Beckman and Koppenol, 1996; Zamora et al., 2000). Three different isoforms are involved in the production of Nitric oxide in various parts of the body. Neuronal NOS (nNOS, ${ }^{3}$ NOS1) is produced by both autonomic and peripheral nervous system to serve as a neurotransmitter and to support inter and intra neuronal communication. Chromosomes 12 carry the gene coding for nNOS. While, endothelial NOS (eNOS) and inducible NOS (iNOS, $\mathrm{NOS}_{2}$ ) is constitutively expressed isoenzyme principally produced from endothelial cells. Chromosome 7 and 17 are responsible for carrying an encoded gene for the iNOS and eNOS respectively. Elevated intracellular $\mathrm{Ca}^{2}$ levels are required for the activation of both enzymes. But unlike eNOS, iNOS bind with $\mathrm{Ca}^{2+}$ calmodulin more tightly with non-covalent bond hence making the deactivation of iNOS considerably difficult. This leads to the continuous and unstoppable overproduction of iNOS in the body (Alderton et al., 2001; Aktan, 2004; Pautz et al., 2010). Normally, low levels of $\mathrm{NO}$ are essential for the body to regulate and maintain the vascular permeability and homeostasis. However, if the production of $\mathrm{NO}$ exceeds the normal levels then it may lead to the pathogenesis of cardio vascular disorders such as hypertension, heart failure, and atherosclerosis (Cooke and Dzau, 1997; Albrecht et al., 2003). Inflamed human endothelium can contribute to increased iNOS activity up to three- to five-fold beyond its baseline concentrations (Zhang et al., 2007; Brovkovych et al., 2011). Moreover, numerous reports have indicated that eNOS can mimic iNOS activity depending upon the nature and intensity of the stimulus, but more work need to be done to elucidate the mechanism behind this interchangeable behaviors of eNOS (Cirino et al., 2003).

There are several reports in the literature that show that Annonaceae species has a potent iNOS inhibitory activity. Recently Hu et al. (2007) reported that 7-(3,4- dihydroxyphenyl)$\mathrm{N}$-[(4-methoxyphenyl) ethyl] propenamide (Z23) (6) (Figure 1) from $F$. oldhamii exhibited dual action of decreasing the $\mathrm{T}$ cell activation and the production of iNOS in in vivo model of type II bovine collagen induced arthritis. In the light of this study, it was suggested that anti-inflammatory effect of Z23, is through modulating the synthesis of several inflammatory mediators and cytokines involved in the inflammatory process. In conclusion, Z23 has the potential to be a therapeutic option for numerous inflammatory diseases, where the overproduction of NOS and inflammatory cytokines are responsible for development and progression of the disease, e.g., rheumatoid arthritis. Moreover, Adewole and Caxton-Martins (2006) evaluated the corresponding activity of aqueous leave extract of $A$. muricata on streptozotocin induced (STZ)-diabetic rats. The extracts were intra-peritoneally injected to the rats $(100 \mathrm{mg} / \mathrm{kg})$ starting from 5 days after the administration of STZ and stopped on the 30th day of the study period. While control group of rats was injected with same amount of citrate buffer. Histophathological evaluation and bioassay results revealed that $A$. muricata significantly $(p<$ 0.05 ) reduced the glucose and iNOS level in a dose dependent manner. Hence in the light of this study it can be concluded that A. muricata has beneficial effects on pancreatic tissues subjected to STZ-induced oxidative stress. Likewise the recent work of Nhiem et al. (2015) reported three new ent-kaurane diterpenoids and five known isolates from the fruit extract of $A$. glabra and they were evaluated for anti-inflammatory activity. After $24 \mathrm{~h}$ of incubation with the isolates, 7 $\beta, 17$-dihydroxyent-kaur-15-en-19-oic acid 19-O- $\beta$-D-glucopyranoside ester (20) (Figure 1) inhibitory activity was the most significant with the IC $_{50}$ value of $0.01 \mu \mathrm{M}$. Similarly, Shirwaikar et al. (2004) focused on Annona Squamosa, commonly known as custard apple. Several antioxidant-screening models were used to evaluate the free radical scavenging activity of the leaves of A. squamosa. Leave ethanolic extract had the highest scavenging activity against 2,2-azinobis- (3-ethylbenzothiazoline- 6- sulphonate) (ABTS) up to $99.07 \%$ followed by the scavenging of the stable radical 1, 1-diphenyl, 2- picryl hydrazyl (DPPH) (89.77\%), and nitric oxide radical $(73.64 \%)$ at $1,000 \mu \mathrm{g} / \mathrm{ml}$. These findings signify the therapeutic potential of Annona species in traditional medicine.

Xylopia parviflora is a tall tree distributed in East and Central Africa. It is very famous for its traditional uses in coastal regions against stomach disorders, headaches and fever (Nishiyama et al., 2006). Kuate et al. (2011) evaluated the possibility of using $X$. parviflora seed as a food source of natural antioxidant. Several bioassays were performed on water, ethanolic, and hydroethanolic extracts to evaluate the anti-oxidant and free radical scavenging activity. All extracts have shown significant $(p<$ 0.05 ) dose dependent NO inhibition, compared to the control group. However, high test NO inhibition was observed with 
hydo-ethanolic extract, decreasing the iNOS production upto $87 \%$ with $\mathrm{IC}_{50}$ range of $20-50 \mu \mathrm{M}$. The anti-oxidant activity of $X$. parviflora was further supported by the late work of Woguem et al. (2014). This study focused on the essential oils hydro distilled from the fruits of $X$. parviflora. In order to evaluate the anti-oxidant and anti-inflammatory activity of $X$. parviflora, LPS induced raw macrophage 264.7 cell were incubated with essential oils for $24 \mathrm{~h}$. Results suggested that cells treated with $X$. parviflora essential oils have shown decreased NO production in a dose dependent manner exhibiting a potential anti-inflammatory activity.

In recent years, utilization of anti-oxidants has been significantly increased due to its positive role in the management of numerous diseases (Halliwell, 2006). Monodora tenuifolia have already been exploited commercially either as antioxidant additives or nutritional supplements (Pourmorad et al., 2006). To validate the anti-oxidant activity of $M$. tenuifolia Njoku (2007) administered diethyl ether fraction and seed extracts to rats systemically. Since the diazotization of nitrite with sulphanilamide, formation of choromophore followed by coupling with napthethylene diamine served as a marker of NO scavenging activity (Villagra et al., 2007). M. tenuifolia seed extract, pet- ether extract and the diethyl ether fraction were able to inhibit the formation of chormophore in a dose dependent manner. Although, the exact pathway of free radical scavenging activity was not completely understood but it was proposed that activity reported was probably due to the high occurrence of anti-oxidant vitamins and flavonoids. Moreover, no toxicity sign were observed up to a dose level of $5,000 \mathrm{mg} / \mathrm{kg}$ body weight. Moreover, 15 known and three new megastigmane glycosides were isolated from Miliusa balansae and were tested on LPS induced RAW 264.7 macrophage cells (Thao et al., 2015). milbaside A and B (21) and C (22) (Figure 1) were most effective among all isolated compounds, with inhibition values of $98.5 \pm 1.6,90.9 \pm 7.8,84.8 \pm 3.5 \%$, respectively. Rest of the compounds either had weak or failed to show any activity on tested concentration $(10.0,20.0$, and $40.0 \mu \mathrm{M})$.

Polyalthia longifolia var. pendula, commonly known as "Indian Mast Tree" is widely distributed in tropical and subtropical regions. Due to its ability to reduce noise pollution it is extensively cultivated in several Asian countries, specially in Taiwan (Ghosh et al., 2008). In an effort to explore isolates from Taiwanese medicinal plants with anti-inflammatory activity, Wu T. H. et al. (2014) evaluated P. longifolia isolates and fruit extracts for iNOS inhibitory activity. Results from present study suggested that 16-hydroxycleroda-3,13-dien-15,16-olide (23) and 16-oxocleroda-3,13-dien-15-oic acid (24) (Figure 1) has significantly $(P<0.05)$ reduced NO production at $10 \mu \mathrm{g} / \mathrm{mL}$, with 81.1 and $86.3 \%$, inhibition, respectively. Similarly, Saha et al. (2008) conducted a study on Bangladeshi medicinal Plants (Hibiscus mutabilis, Leucas aspera, Ixora coccinea, and P. longifolia). Ethanolic extract of all the tested species showed dose dependent NO direct scavenging activity in following manner L. aspera $>I$. coccinea $>H$. mutabilis $>P$. longifolia. Although, the activity reported for $P$. longifolia was least potential among all the tested extract with $70.67 \%$ with $\mathrm{IC}_{50}$ of $167.08 \mu \mathrm{g} / \mathrm{ml}$ but its inhibitory activity can be considered significant when compared to positive control (ascorbic acid with $74.56 \%$ inhibition). In another account, Johnson et al. (2013) stated that Alphonsea javanica Scheff decreased the expression of eight pro-inflammatory cytokines/enzymes $(0.8-5.0 \mu \mathrm{M})$ including iNOS. Then again, Aquila et al. (2009) reported similar activity of berenjenol (7) (Figure 1), isolated from Oxandra cf. xylopioides. The anti-inflammatory activity was assessed on sub chronic inflammation induced by repetitive application of 12-O-tetradecanoyl-phorbol-13-acetate (57\% inhibition, $7 \times 1$ $\mu \mathrm{mol} /$ ear). While it reduced the expression of iNOS by $80 \%$ at $50 \mu \mathrm{M}$. Hence suggesting that these species can be used as potent and novel therapeutic agent for scavenging of $\mathrm{NO}$ and the regulation of pathological conditions caused by excessive generation of NO and by product.

\section{Reactive Oxygen Species (ROS) Inhibition}

ROS are usually characterized as incompletely reduced metabolites of oxygen that have potent oxidizing potentials (Casteilla et al., 2001; Mittal et al., 2014). ROS actions can be variant depending upon their concentrations. At high concentrations, ROS are injurious to body but at low concentrations, ROS serve as intricate signaling functions (Taniyama and Griendling, 2003; DelloStritto et al., 2016). Under normal circumstances, the human body produces numerous antioxidants such as catalase and glutathione peroxidase, in order to balance out the deleterious effects of ROS. However, in certain inflammatory conditions, this balance is disturbed due to the excessive generation of ROS (Drake et al., 1998; Cominelli, 2004; Reuter et al., 2010). In other way, neutrophils produce a large quantity of ROS at the site of inflammation in order to fight against the foreign bodies. As a result lipid peroxides are produced which gives rise to pathophysiological changes associated with an oxidative stress (Wolfreys and Oliveira, 1997; Dabrowski et al., 1999; Aprioku, 2013). This physiological response is known as oxidative burst. It provides support to host defense, but it can also result in collateral destruction of host tissues (Chen and Junger, 2012). Henceforth, suppression of the excessive pathophysiological activation of neutrophils can be used to treat inflammatory diseases. A few studies including Barreca et al. (2011) evaluated the antioxidant activity of ethanolic, methanolic, and dimethyl formammide extracts of Annona cherimola on hydrogen peroxide induced lymphocytes. Although, all extracts showed significant antioxidant and ferric reducing potential but highest scavenging activity was reported for dimethyl formammide extract against DPPH, ABTS and $\mathrm{O}_{2}^{-}$. Whereas ethanolic extract was reported to have highest activity against tert-butyl hydroperoxide induced lipid peroxidation. Invariably in the light of the fact that various antioxidants also possess antimutagenic activity, Ravikumar et al. (2008) focused on screening antimutagenic and antioxidant activity of Polyalthiam cerasoides. Antioxidant activity was evaluated based on inhibitory activity on hydroxyl radical, superoxide radical, $\mathrm{DPPH}$ free radical scavenging and $\mathrm{Fe}^{3+}$ reducing properties. Results suggested that methanolic extract inhibited DPPH and superoxide anion in a dose dependent manner. Moreover, similar activity was reported against hydroxyl radicals produced by the reaction of $\mathrm{Fe}^{3+}$-EDTA together with $\mathrm{H}_{2} \mathrm{O}_{2}$ and ascorbic 
acid. In addition, by reducing $\mathrm{Fe}^{3+}$ to $\mathrm{Fe}^{2+}$ ions the methanolic extract had exhibited strong reducing potential. These results clearly indicate that methanolic extract of $P$. cerasoides have powerful anti-oxidant and reducing potential. Likewise, Hwang et al. (2009) reported similar activity of isopedicin, a flavanone derived from F. oldhamii. Results suggested that isopedicin decreased superoxide anion production in FMLP stimulated RAW macrophage with an $\mathrm{IC}_{50}$ value of $0.34 \pm 0.03 \mu \mathrm{M}$. In addition, phosphodiesterase inhibition enhanced the activity of PKA and cAMP. Moreover, FMLP induced kinase and c-Jun N-terminal kinase phosphorylation was inhibited by isopedicin. However, isopedicin was unable to reverse the FMLP induced calcium mobilization and p38 mitogen activated protein kinase phosphorylation. Hence it is proposed that antioxidant activity of isopedicin is due to the elevation of cellular cAMP and activation of PKA through its inhibition of cAMP-specific PDE. Numerous other species of Annonaceae can produce similar super oxide anion generation and elastrase inhibitory activity in micro-molar concentration. For instance, Chang et al. (2006) isolated a novel clerodane diterpenoid 16hydroxycleroda-13-ene-15,16-olide-3-one (25) (Figure 1) along with other known 23 compounds from the methanolic extract of $P$. longifolia leaves. Anti-inflammatory activities of isolated compounds were evaluated on formyl-L-methionyl- $L$-leucyl- $L$ phenylalanine/cytochalasin B (FMLB/CB) stimulated superoxide generation in neutrophils. 16-oxocleroda-3,13E-dien-15-oic acid methyl ester (26) and 16-hydroxycleroda-3, 13(14)E-dien-15-oic acid (27) (Figure 1) exhibited significant inhibitory activity against both models with $\mathrm{IC}_{50}$ value of $0.6 \pm 0.09$ and 1.49 $\pm 0.28 \mu \mathrm{g} / \mathrm{mL}$, respectively. In continuation of previously mentioned work by Liou et al. (2014), member of same research group investigated phyto constituents from the leaves of Polyalthia parviflora. Parvistones (28) (Figure 1), a styryllactones and two $6 \mathrm{~S}$ configuration derivative inhibited fMLP/CB-induced superoxide anion generation and elastase release. Likewise, Chan et al. (2013) isolated a new melodamide A(29) (Figure 1) phenolic amide along with 12 known compounds from the methanolic leave extract of Melodorum fruticosum. Melodamide A (29) has shown significant inhibitory activity with $I_{50}$ value of $5.19 \mu \mathrm{M}$ against both $\mathrm{O}^{-2}$ and elastase. However, synthetic derivative of melodamide $\mathrm{A}(29)$ with a 2-bromo substitution on ring A failed to show any substantial anti-oxidant activity. Similarly, in recent work of Hsu et al. (2016) seven compounds including 3-methyl-4, 5-dihydro-oxepine (flexuvaroxepine A) (30) (Figure 1), four polyoxygenated cyclohexene and two polyoxygenated cyclohexene derivatives, together with four known flavones were isolated from methanolic extract of Uvaria flexuosa. All isolated compound were evaluated against superoxide anion generation and elastase release. Out of all the isolated compounds flexuvarol B (31) and chrysin (8) (Figure 1) have shown significant inhibitory activity against elastase release and superoxide anion generation with $\mathrm{IC}_{50}$ of $2.25-5.55 \mu \mathrm{M}$. Similarly, Njoku (2007) reported anti-oxidant activity for the seed extract of M. tenuifolia. He reported that seed extracts were able to inhibit lipid peroxidation and free radical generation in liver homogenate in a dose dependent manner.

\section{Suppressing the Transmigration and Phagocytosis of Mono Nuclear Cells}

More than few reports have called attention to other mechanism related with the flavonoids of A. dioica. This study focused on the several aspects including anti-proliferative, antioxidant, and anti-inflammatory activity of $A$. dioica (Formagio et al., 2013a). DPPH assay was employed in order to evaluate the free radical scavenging activity of four fractions including hexane, chloroform, ethyl acetate and hydromethanol fraction. Furthermore carrageenan induced paw edema test was used for the further evaluation. Results suggested that ethyl acetate and hydromethanol fractions were most potent among all the tested fractions with an $\mathrm{IC}_{50}$ of 8.53 and $10.57 \mu \mathrm{g} / \mathrm{mL}$, respectively. Whereas, methanolic extract was able to significantly reduce the carrageenan-induced edema in dose and time dependent manner $(30-300 \mathrm{mg} / \mathrm{kg})$. It was concluded that A. dioica St. and several other species of Annonacea family have a unique mechanism of suppressing the transmigration and phagocytosis by polymorphonuclear leukocytes (PMNs) and helps in the alleviation of unnecessary and deleterious production of ROS, hyperalgesia, and other classical symptoms of inflammation. In succession of his previous work, Formagio et al. (2013b) further evaluated the essential oils, hydrodistillated form the leaves of Annona sylvatica. Carrageenan induced paw edema test was employed to assess the anti-inflammatory activity. Upon oral administration of essential oils, potent anti-inflammatory and antioxidant activity was reported. It was proposed that the presence of z-caryophyllene (44) and $\beta$-maliene (46) (Figure 1) in essential oil was responsible for potential anti-oxidant activity. Moreover, several other phytochemical classes including chalcone have been reported to share similar activity. Somsrisa et al. (2013) managed to isolated one new dihydrochalcone derivative $4^{\prime}, 6^{\prime}$-dihydroxy2',4-dimethoxy-5'-(2"'-hydroxybenzyl)dihydrochalcone and one known dihydrochalcone, 4', 6'-dihydroxy-2',4dimethoxydihydrochalcone (32) (Figure 1) from the twig and leaves of Cyathostemma argenteum. Ethyl phenylpropiolate was applied tropically on the inner and outer part of the ear of rats to induce ear edema. Dose of $1 \mathrm{mg} /$ ear test compounds were also applied tropically just before the application of irritant to evaluate their analgesic activity. A significant time dependent inhibition was observed for both isolated compounds at a dose of $1 \mathrm{mg} / \mathrm{ear}$. Hence concluding that both compounds are very effective in an acute phase of inflammation and has the ability to antagonize or decrease the vascular permeability of inflammatory mediators including histamine, serotonin, bradykinin, and prostaglandin (PGs). Chavan et al. (2012) reported similar activity for a diterpines, kaur-16-en-19-oic acid (34) (Figure 1) isolated from the bark of Annona reticulate. Hot plate method was employed to assess the analgesic activity. Whereas anti-inflammatory activity was evaluated using carrageenan induced rat paw edema assay. Significant analgesic and anti-inflammatory activity was reported for kaur-16-en-19-oic acid (34), at doses of 10 and $20 \mathrm{mg} / \mathrm{kg}$. This study points out a lead antiinflammatory compound, which should be further explored for therapeutics. 
In the last few decades exceptional progress has been made for the development of new anti-inflammatory and analgesic drugs. However, we are far behind from finding an ideal class of drug with maximum efficacy and minimum side effects. As a part of this effort Vendramini-Costa et al. (2014) evaluated a styryllacton, goniothalamin (1) (Figure 1) widely distributed among the genus Goniothalamus. Results suggested that Goniothalamus has significantly reduced the carrageenan induced paw edema in mice. Moreover, effective concentrations were also evaluated for its potential toxicity and results suggested that no sign of toxicity was observed at effective concentrations. Moreover, Meiocarpidium lepidotum has exhibited significant analgesic and anti-inflammatory activity in mice and rat (Meddah et al., 2013). Tail flick tests, acetic acid- induced writhing, carrageenaninduced hyperalgesia in mice were employed for the evaluation of anti-inflammatory activity. Paw edema was significantly reduced after the administration of extract. Moreover, acetic acid induced writhings and tail-flicks were significantly $(p<$ 0.001 ) reduced at $1 \mathrm{mg} / \mathrm{kg}$ dose. Hence, proving a very potent analgesic and anti-inflammatory activity at small concentrations. Moreover, the intra-peritonial injection of methanolic fruit extract of $D$. chrysocarpa in rat model have shown significant analgesic and anti-inflammatory activity in a dose dependent manner (Almeida et al., 2012). At dose range of 100, 200, and $400 \mathrm{mg} / \mathrm{kg}$ acetic-acid-induced abdominal writhes were significantly reduced. Moreover, significant results were also recorded for hot-plate test and formalin test. It was proposed that corresponding activity reported is due to its dual action on peripheral and central nervous system. Additionally, Ishola et al. (2016) reported similar activity for hydro-ethanolic seed extract of Monodora myristica. It completely inhibited the xylene induced ear edema. Significant increase in threshold and decrease in acetic induce abdominal writings were observed in a dose dependent manner at concentration range of $50-200 \mathrm{mg} / \mathrm{kg}$. Hence, this study provides scientific evidence for the use of M. myristica in traditional medicine for pain management. In the latest work of Popoola et al. (2016) focused three plant species commonly used for their indigenous anti-cancer activity. In this present study anti-oxidative and anti-inflammatory activity of Garcinia kola Heckel (stem bark), Uvaria chamae (root), and Olax subscorpioidea (root) were evaluated using in vivo inflammatory models. Formaldehyde and carrageenan induced rat paw edema was significantly reduced by all three species, in a time dependent manner. Maximum inhibitory activity was observed at $400 \mathrm{mg} / \mathrm{kg}$ when compared with the reference drugs. Thus, this study provides some scientific evidence of the usage of these three species in traditional anticancer and anti-inflammatory regimens. Seangphakdee et al. (2013) reported similar anti-inflammatory activity for polyoxygenated cyclohexane zeylenol (35) (Figure 1) isolated from Uvaria grandiflora. Anti-inflammatory activity of zeylenol (35) was evaluated on rats using ear edema assay. Zeylenol (35) reduced the ear edema in a time dependent manner; activity recorded was equivalent to positive control, phenylbutazone. Results suggested that the test compound is effective against acute phase inflammation and able to inhibit the synthesis or release of various inflammatory mediators (histamine, serotonin, bradikinine, prostaglandin) responsible for producing vasodilation. Moreover, the mice treated with essential oil with the leave of Xylopia laevigata have also significantly $(P<0.05$ and $P<0.001)$ reduced formalin induced abdominal writhing (Queiroz et al., 2014). Whereas, oral administration of essential oils decreased carrageenan-induced peritonitis and paws edema. Hence providing the scientific evidence to the traditional use of $X$. laevigata as anti-inflammatory remedy.

\section{Pro-Inflammatory Cytokines Inhibition}

According to Bulua et al. (2011) "mitochondrial ROS (mtROS) act as signaling elements to induce pro-inflammatory cytokine production" (Nakahira et al., 2011; Zhou et al., 2011). This self-explanatory statement highlights the role of ROS in the production of pro-inflammatory cytokines. TNF- $\alpha$ is a major cytokine, responsible for inducing various other proinflammatory cytokines. Using its pyrogic activity TNF- $\alpha$ can induce mononuclear cells to produce inflammatory mediators like iNOS and ROS. These inflammatory mediators can further induce the production of TNF- $\alpha$ simultaneously. This leads to the production of IL-1 and IL- 6 and proinflammatory cytokines and chemokines by activation of NF-кB (De Simone et al., 2015; Chen et al., 2016). Tumor necrosis factor alpha (TNF- $\alpha$ ) and interleukin-6 (IL-6) are pro-inflammatory cytokines released by stimulated macrophages to augment the inflammatory response and injure cells and its surroundings (Whiteley et al., 2009; Olefsky and Glass, 2010). Hence decreasing the TNF- $\alpha$ and IL6 levels may repress tissue injury caused by the inflammation. For instance fruit and seed extract from A. squamosa decreased TNF- $\alpha$ and IL- 6 levels in LPS-stimulated macrophages (Yang et al., 2002). Similar activity was reported by Chuang et al. (2008) for Annona montana. The study was conducted to evaluate four new cyclomontanins isolated from the methanolic extract of $A$. montana seeds. LPS induced murine macrophage J774A.1 cells were used to evaluate the cytokines production inhibitory activity of cyclomontanins. Results suggest that maximum TNF- $\alpha$ and IL-6 inhibitory activity was reported for cyclomontanin A (36) and cyclomontanin C (37) (Figure 1). Although, cyclomontanin D (38) and annomuricatin C (37) (Figure 1) inhibitory activity is at relatively higher concentration $(30 \mu \mathrm{g} / \mathrm{mL})$. However, upon stimulating the cells with Pam3Cys cyclomontanin D exhibited dose dependent inhibition at various concentrations 3, 5, 10 , 30 , and $50 \mu \mathrm{g} / \mathrm{mL}$. These results demonstrated a potent antiinflammatory activity of synthetic analogs of cyclopeptides but lacks accuracy to pin point the exact mechanism of inhibition. Dellai et al. (2010) reported similar activity of cyclic peptides isolated from the seeds of A. squamosa. cyclosquamosin D (39) and met-cherimolacyclopeptide B (40) (Figure 1) and their analogs were evaluated using well established enzyme-linked immunosorbent assay (ELISA). Fourteen synthetic analogs were prepared from cyclosquamosin D (39) (Figure 1), few of them failed to show any bioactivity and hence they were included as a negative control. Whereas, few synthetic compounds were able to show superior activity then the natural products. Three analogs were able to suppress the IL6 and TNF $\alpha$ equally. Moreover, no activity was reported for the natural cyclic peptide cherimolacyclopeptide $B$ and met-cherimolacyclopeptide. Whereas, their analogs were able to significantly reduce the production of TNF-a and IL-6 
in LPS induced macrophage cells. Two new cyclic peptides, fanlizhicyclopeptide A (41) and fanlizhicyclopeptide B (42) (Figure 1) isolated from the fruit of A. squamosa reported similar activity (Wu P. et al., 2014). Fanlizhicyclopeptide A (41) and fanlizhicyclopeptide $\mathrm{B}(42)$ (Figure 1) were able to reduce the secretion of TNF $\alpha$ by 32 and $27 \%$. However, IL- 6 activity was more pronounced then TNF $\alpha$ with 51 and 57\% inhibition. Few years later $\mathrm{Ge}$ et al. (2013) demonstrated that aristololactam (43) (Figure 1) an alkaloid extracted from F. oldhamii against TNF-a and IL-6. From the results, the ethanol extract and its $\mathrm{CHCl}_{3}, \mathrm{EtOAc}$, and $\mathrm{n}-\mathrm{BuOH}$-soluble parts showed significant inhibitory effects against LPS-induced IL-6 production, and the $\mathrm{CHCl}_{3}$-soluble part inhibited TNF- $\alpha$ production. As mentioned before $7^{\prime}$-(3', $4^{\prime}$-dihydroxyphenyl)-n-[(4-methoxyphenyl) ethyl] propenamide (Z23) (6) (Figure 1) from F. oldhamii, have shown significant inhibitory activity against nitric oxide synthase (iNOS) and cyclooxygenase 2 (COX2). In the same study, $\mathrm{Hu}$ et al. (2008) reported that Z23 was able to produce more pronounce effect on TNF- $\alpha$ then IL-6. Moreover, in recent study of Vendramini-Costa et al. (2017) evaluated the cytokines inhibitor activity of goniothalamin (1) in colitis-associated cancer (CAC) and dextran sulfate sodium (DSS) induced-colitis mice model. Results indicate that goniothalamin decreased the gene expression of IL $1 \beta$, TNF- $\alpha$, IL-6, IL-23A, IL-22, and IL17A. Moreover, IL-6, IL-17, and TNF- $\alpha$ production was also significantly reduced in tumor tissue. It was concluded from this data that GTN could be a potential candidate for the treatment of progression and development of colon rectal cancer due to its potent anti-inflammatory activity. Rojano et al. (2007) indicated one new and unusual cycloartane triterpene, berenjenol (1) (Figure 1) and its three synthetic derivative isolated from the leaves of Oxandra xylopioides. Test compounds were coincubated with LPS induced RAW 264.7 macrophages. Whereas, the inhibitory activity of the test compound was evaluated using ELISA kit. Isoespintanol (Figure 1), a synthetic derivative of berenjenol (1) had decreased IL-1 production by $72 \%$ at $100 \mu \mathrm{M}$ and reduced IL-1 mRNA synthesis.

In very interesting study conducted by López et al. (2009), reported anti leishmanial and anti-inflammatory activity of eight extracts and essential oil from the leaves and seeds of Xylopia discrete. Results from this study suggested that Xylopia discrete failed to produce any significant effect on IL-10 IL-12 and TNF $\alpha$. However, rise in level of monocyte chemotactic protein 1 (MCP-1) was observed in infected macrophages. This rise of MCP-1 caused to decrease the number of Leishmania parasites in infected cells. The proposed mechanism involves modulating the levels of MCP-1, which is responsible for the prophylactic function in maintaining cytokines level (Brandonisio et al., 2002; Dey et al., 2007). Usually, macrophage produce proinflammatory cytokines plus MCP-1 to induces the production of IL-12 and inhibiting IL-10 and TGF- $\beta$. Th1 phenotype was provoked in response to treatment with MCP-1, macrophage inflammatory protein supports prophylactic role to these chemokines to control of Leishmania parasites. This study suggests that $X$. discreta possesses antileishmanicidal potential due to its immunomodulatory activity. But more work need to be done in vivo models in order to confirm these bioactivities.

\section{CLINICAL TRIALS}

Currently, only one double-blind randomized, placebocontrolled, Phase 0 trial clinical trial has been carried out to highlight the anti-inflammatory effect of Annonaceae sp. http://www.clinicaltrials.gov/. The purpose of this study was to evaluate A. muricata for its nutritional status, role in improving the quality of life, and effect on fecal butyrate, inflammation, and colorectal cancer cells. The leaves of A. muricata were focused in this study due to the presences of biologically active polyphenols and acetogenins with reported anti-inflammatory and anti-cancer activity. Moreover, this was an effort to validate the previous findings in in vitro, animal study and traditional uses of this specie. Thirty patients were involved in this study. Both genders were included with the age of 18 years and above. Patients with satisfactory hematological reports and Karnofsky performance $>60 \%$ were taken into the study. Two patient groups were formed; one group was treated with crude ethanolic extract of A. muricata while other group served as a control group (maltose treated group). After every fortnight patient's dietary intakes were assessed. Whereas, the hematological reports, fecal butyrate level, nutritional status, and markers of system systemic inflammation of the patients were assessed in the beginning and the end of the study. A. muricata ethanol extract were administered with $300 \mathrm{mg} /$ day dose, whereas equal amount of cellulose was administered to control group for 8 weeks to evaluate anti-inflammation and anti-proliferative activity. Whereas, the ethanol-soluble fraction of water extract was used along placebo to evaluate the nutritional value of $A$. muricata. Primary outcome measures after 8 weeks of the study concluded that $A$. muricata has nutritional status and can be used for as a dietary supplement to improve the quality of Life. Secondary outcome measures stated that extract of A. muricata did not show any cytotoxic activities in MTT assay using colorectal cells when exposed to patient serum for $48 \mathrm{~h}$. Nevertheless, no data was provided regarding anti-inflammatory activity of the extract, neither material nor methods were discussed in detail. Therefore, this interesting study may lead to insightful development of knowledge regarding its clinical efficacy. Since the number of patients participated in this clinical trials were insufficient and duration of study should be more than 8 weeks, and results of parameters under investigation were not discussed properly and left open-ended. Nonetheless, more randomized controlled trials are required to cover additional parameters to draw fruitful conclusion about A. muricata and other Annonacaee species.

\section{TOXICOLOGY}

Numerous studies have been conducted to explore the pharmacological properties of Annonaceae sp. While the toxicological aspects associated with the use of these species have been ignored significantly. However, according to toxicological studies the frequent use of few Annonaceae species has been associated with potentially hazardous side effects. For instance, Lannuzel et al. (2002) carried out the study to evaluate the abnormally high prevalence of levodopa-resistant Parkinsonism in West Indies. Some proposed that frequent 
consumption of fruit of $A$. muricata is the reason behind this atypical Parkinsonism. To validate this claim mesencephalic dopaminergic neurons were exposed to alkaloids and root and bark extracts (totum) of $A$. muricata for $24 \mathrm{~h}$. After $24 \mathrm{~h}$ it was observed that $50 \%$ of dopaminergic neurons were deteriorated with, $4.3 \mu \mathrm{g} / \mathrm{ml}(13 \mu \mathrm{M})$ coreximine, or $100 \mu \mathrm{g} / \mathrm{ml}(304 \mu \mathrm{M})$ reticuline and $18 \mu \mathrm{g} / \mathrm{ml}$ totum. Microscopic visualization of dead neuron revealed DNA fragmentation, purposing apoptosis to be the possible cause of death. A few years later Champy et al. (2004) shared the similar concern toward the inhabitants of Guadeloupe island. He proposed that atypical Parkinsonism is prevalent in this region due to frequent consumption of the annonaceous acetogenins (lipophilic complex I inhibitors) present in $A$. muricata. To elucidate this claim, high concentrations of annonacin (3.8 and $7.6 \mathrm{mg}$ per $\mathrm{kg}$ per day for 28 days) were intravenously administered to rats. Annonacin inhibited brain homogenates complex 1 in concentrations dependent manner and ATP level inside brain was reduced by $44 \%$. Although, no systemic toxicity was observed but neuro-pathological abnormalities were observed in the basal ganglia and brainstem nuclei. Significant loss of dopaminegernic $(-31.7 \%)$, cholinergic $(-37.9 \%)$, GABAergic neurons $(-39.3 \%)$ in the stratum was accompanied by increase numbers of astrocytes cell (35.4\%). Present findings are enough to support the hypothesis that annonacin may be involved in the Guadeloupean Parkinsonism and bolster the theory that ecological toxins may instigate atypical Parkinsonism. Lannuzel et al. (2003) reported annonacin to be more toxic than 1-methyl-4-phenylpyridinium $\left(\mathrm{MPP}^{+}\right)$ $\left(\mathrm{EC}_{50} 0.018\right.$ vs. $1.9 \mu \mathrm{M}$ ) in dopaminergic neuronal dysfunction. It was proposed that Annonacin interfares with the energy production mechanism of neuronal cells hence leading to the dopaminergic neuronal cell death. These studies conclude that these species may alter functions of dopaminergic nerve cells in vitro. It is in this way possible that $A$. muricata could bring about the neuronal dysfunction and neuro degenerative diseases upon frequent use (Caparros-Lefebvre and Lees, 2005; Ludolph et al., 2009).

A. congensis bark and X. aethiopica fruits have been extensively used in the treatment of diabetes and due to its extensive use in tropical regions Ogbonnia et al. (2008) designed this study to evaluated the acute and subacute toxicity of a water: alcoholic extract $(1: 1)$ on Swiss albino rats. Animals were fed with various concentrations ranging from 1 to $20 \mathrm{~g} / \mathrm{kg}$ body weight for 30 days. Significant amount of weight gain was observed at low doses but there were no signs of drug induced toxicity or animal death at these concentrations and. However, sub-acute toxicity study have shown signs of renal toxicity. Moreover, an acute and sub-acute toxicity study was conducted on the aqueous stembark extract of E. chlorantha (Tan et al., 2007). Acute toxicity was evaluated by administering single oral dose of 1,000, 3,000, and $5,000 \mathrm{mg} / \mathrm{kg}$ of plant extract to rats and monitoring for any signs of growth impairment and death for 7 consecutive days. Sub-acute toxicity was assessed by evaluation of hematological and biochemical parameters after administrating 250, 500, and $1,000 \mathrm{mg} / \mathrm{kg}$ extract for 42 consecutive days. Rats were sacrificed on 42nd day to study the histological analysis of vital organs including heart, lungs, liver, kidney. At acute doses no death, drug induced symptoms or growth impairment was observed. Whereas, sub-acute toxicity study presented histopathological signs in the liver, lungs, and kidneys at dose of $1,000 \mathrm{mg} / \mathrm{kg}$. Moreover, significant $(P<0.05)$ increase in values of ALT, AST, and platelet counts were also observed. Hence concluding that the E. chlorantha extract exhibit no acute toxicity up to $5,000 \mathrm{mg} / \mathrm{kg}$, but can cause lung, hepatic and kidney disorders at doses $>500 \mathrm{mg} / \mathrm{kg}$. Moody et al. (2007) in his recent study evaluate the acute and sub-chronic toxicity of E. chlorantha. Ethanolic extracts were administered to Swiss rats through oral and intra-peritoneal route of administration. Upon histopathological examination, no pathological signs were observed on any organ expect for lungs, which exhibited mild to moderate edema upon examination.

\section{CONCLUSIONS AND FUTURE DIRECTIONS}

This review is an effort to abridge the ethnobotany, morphology, phytochemistry, and particularly focusing on the antiinflammatory activity of the Annonaceae species. It additionally conveys insights of the Annonaceae family, which may contribute toward highlighting its isolated compounds as a future candidate for drug discovery. A careful review of the literature has shown that few studies have proposed the scientific evidence for the traditional uses of Annonaceae sp., its pleotropic therapeutic activities including analgesic, anti-pyretic, anti-ulcer, anti-hypertensive, oral hypoglycemic and wound healing. Sesquiterpenes and diterpenes from Annonaceae have shown promising anti-inflammatory activity. Hence making this class of drug potential clinical trial candidates in anti-inflammatory therapy. Majority pharmacological studies have supported their medicinal use of Annonaceae family in traditional medicine against pain (Badrie and Schauss, 2010; Cercato et al., 2015), anthelmintic (Auddy et al., 2003; Bhalke and Chavan, 2011), malaria (Duke, 2000; Garavito et al., 2006), and weight loss (Cercato et al., 2015). Further studies should be carried out to elucidate the exact composition of plant extracts to standardize the formulations based on ingredients. The randomized human trial should be conducted with compounds with superior IC50 to convert their pre-clinical results into clinical data. This will help us to develop a better understanding of the pharmacokinetics and dynamics, bioavailability, and toxicity associated with their use in clinical setting.

\section{AUTHOR CONTRIBUTIONS}

All authors listed have made a substantial, direct and intellectual contribution to the work, and approved it for publication.

\section{ACKNOWLEDGMENTS}

The authors would like to thank the Ministry of Agriculture and Agro-based Industry (MOA) Malaysia, for the financial support under grant number NH0413D016. Moreover we like to thank Universiti Kebangsaan Malaysia (UKM) for allowing us to use all its facilities. 


\section{REFERENCES}

Adewole, S. O., and Caxton-Martins, E. A. (2006). Morphological changes and hypoglycemic effects of Annona muricata linn. (annonaceae) leaf aqueous extract on pancreatic $\beta$-cells of streptozotocin-treated diabetic rats. Afr. J. Biomed. Res. 9, 173-180. doi: 10.4314/ajbr.v9i3.48903

Akaberi, M., Iranshahy, M., and Iranshahi, M. (2015). Review of the traditional uses, phytochemistry, pharmacology and toxicology of giant fennel (Ferula communis L. subsp. communis). Iran. J. Basic Med. Sci. 18, 1050-1062. doi: $10.22038 / \mathrm{ijbms} .2015 .6042$

Akira, S., Uematsu, S., and Takeuchi, O. (2006). Pathogen recognition and innate immunity. Cell 124, 783-801. doi: 10.1016/j.cell.2006.02.015

Aktan, F. (2004). iNOS-mediated nitric oxide production and its regulation. Life Sci. 75, 639-653. doi: 10.1016/j.lfs.2003.10.042

Albrecht, E. W., Stegeman, C. A., Heeringa, P., Henning, R. H., and van Goor, H. (2003). Protective role of endothelial nitric oxide synthase. J. Pathol. 199, 8-17. doi: $10.1002 /$ path. 1250

Alderton, W. K., Cooper, C. E., and Knowles, R. G. (2001). Nitric oxide synthases: structure, function and inhibition. Biochem. J. 357, 593-615. doi: $10.1042 / b j 3570593$

Al-Dhubiab, B. E. (2012). Pharmaceutical applications and phytochemical profile of Cinnamomum burmannii. Pharmacogn. Rev. 6, 125-131. doi: 10.4103/0973-7847.99946

Almeida, J., Oliveira, M., Guimarães, A., Oliveira, A., Ribeiro, L., Lúcio, A., et al. (2011). Phenolic quantification and antioxidant activity of Anaxagorea dolichocarpa and Duguetia chrysocarpa (Annonaceae). Int. J. Pharmacol. Biol. Sci. 2, 367-374.

Almeida, J. R., Araújo, E. C., Ribeiro, L. A., de Lima, J. T., Nunes, X. P., Lúcio, A. S., et al. (2012). Antinociceptive activity of ethanol extract from Duguetia chrysocarpa Maas (Annonaceae). ScientificWorldJournal 2012:859210. doi: 10.1100/2012/859210

Annan, K., Dickson, R., Sarpong, K., Asare, C., Amponsah, K., and Woode, E. (2013). Antipyretic activity of Polyalthia longifolia Benth. \& Hook. F. var. pendula (Annonaceae), on lipopolysaccharide-induced fever in rats. J. Med. Biomed. Sci. 2, 8-12.

Aprioku, J. S. (2013). Pharmacology of free radicals and the impact of reactive oxygen species on the testis. J. Reprod. Infertil. 14, 158-172.

Aquila, S., Rojano, B., Recio, M. C., Giner, R. M., Schinella, G. R., Debenedetti, S. L., et al. (2009). Anti-inflammatory activity of berenjenol and related compounds. Planta Med. 75, 18-23. doi: 10.1055/s-0028-10 88343

Araujo, C. D. S., de Oliveira, A. P., de Oliveira-Junior, R. G., de Siqueira-Filho, J. A., Braz-Filho, R., Tavares, J. F., et al. (2017). Chemical constituents isolated from extracts of Annona vepretorum Mart.(Annonaceae) leaves. J. Med. Plants Res. 11, 439-444. doi: 10.5897/JMPR2017.6385

Arribas-Gómez, I., Duque-Marín, I., Pérez de Lema, G., Díez-Marqués, M. L., Lucio-Cazaña, J., Rodríguez-Puyol, M., et al. (1995). A possible role for platelet-activating factor in the hydrogen peroxide-induced TXB2 and PGE2 glomerular synthesis. J. Lipid Res. 36, 260-265.

Arya, A., Taha, H., Khan, A. K., Shahid, N., Ali, H. M., and Mohd, M. A. (2014). In vivo antidiabetic and antioxidant potential of Pseudovaria macrophylla extract. In Vivo 1:12490.

Auddy, B., Ferreira, M., Blasina, F., Lafon, L., Arredondo, F., Dajas, F., et al. (2003). Screening of antioxidant activity of three Indian medicinal plants, traditionally used for the management of neurodegenerative diseases. J. Ethnopharmacol. 84, 131-138. doi: 10.1016/S0378-8741(02)00322-7

Badr, K. F., DeBoer, D. K., Takahashi, K., Harris, R. C., Fogo, A., and Jacobson, H. R. (1989). Glomerular responses to platelet-activating factor in the rat: role of thromboxane A2. Am. J. Physiol. 256(1 Pt 2), F35-43.

Badrie, N., and Schauss, A. (2010). "Soursop (Annona muricata L.): composition, nutritional value, medicinal uses, and toxicology," in Bioactive Foods in Promoting Health: Fruits and Vegetables, eds R. R. Watson and V. R. Preedy (Oxford: Elsevier Inc.), 621-643.

Baeuerle, P. A., and Baltimore, D. (1996). NF-к B: ten years after. Cell 87, 13-20. doi: $10.1016 /$ S0092-8674(00)81318-5

Baillon, H. (1868). Anonaceae mexicanae liebmannianae enumeratae. Adansonia $8,265-269$.
Barnes, P. J., and Karin, M. (1997). Nuclear factor-kappaB: a pivotal transcription factor in chronic inflammatory diseases. N. Engl. J. Med. 336, 1066-1071. doi: 10.1056/NEJM199704103361506

Barreca, D., Lagana, G., Ficarra, S., Tellone, E., Leuzzi, U., Galtieri, A., et al. (2011). Evaluation of the antioxidant and cytoprotective properties of the exotic fruit Annona cherimola Mill.(Annonaceae). Food Res. Int. 44, 2302-2310. doi: 10.1016/j.foodres.2011.02.031

Beckman, J. S., and Koppenol, W. H. (1996). Nitric oxide, superoxide, and peroxynitrite: the good, the bad, and ugly. Am. J. Physiol. Cell Physiol. 271, C1424-C1437.

Bele, M. Y., Focho, D. A., Egbe, E. A., and Chuyong, B. G. (2011). Ethnobotanical survey of the uses Annonaceae around mount Cameroon. Afr. J. Plant Sci. 5, 237-247.

Bhalke, R. D., and Chavan, M. J. (2011). Analgesic and CNS depressant activities of extracts of Annona reticulata Linn. bark. Phytopharmacology 1, 160-165.

Block, M. L., Zecca, L., and Hong, J. S. (2007). Microglia-mediated neurotoxicity: uncovering the molecular mechanisms. Nat. Rev. Neurosci. 8, 57-69. doi: $10.1038 / \mathrm{nrn} 2038$

Brandonisio, O., Panaro, M. A., Fumarola, I., Sisto, M., Leogrande, D., Acquafredda, A., et al. (2002). Macrophage chemotactic protein-1 and macrophage inflammatory protein-1 alpha induce nitric oxide release and enhance parasite killing in Leishmania infantum-infected human macrophages. Clin. Exp. Med. 2, 125-129. doi: 10.1007/s102380200017

Broide, D. H., Lawrence, T., Doherty, T., Cho, J. Y., Miller, M., McElwain, K., et al. (2005). Allergen-induced peribronchial fibrosis and mucus production mediated by IкB kinase $\beta$-dependent genes in airway epithelium. Proc. Natl. Acad. Sci. U.S.A. 102, 17723-17728. doi: 10.1073/pnas.0509235102

Brovkovych, V., Zhang, Y., Brovkovych, S., Minshall, R. D., and Skidgel, R. A. (2011). A novel pathway for receptor-mediated post-translational activation of inducible nitric oxide synthase. J. Cell. Mol. Med. 15, 258-269. doi: 10.1111/j.1582-4934.2009.00992.x

Bulua, A. C., Simon, A., Maddipati, R., Pelletier, M., Park, H., Kim, K. Y., et al. (2011). Mitochondrial reactive oxygen species promote production of proinflammatory cytokines and are elevated in TNFR1-associated periodic syndrome (TRAPS). J. Exp. Med. 208, 519-533. doi: 10.1084/jem.20102049

Buncharoen, W., Saenphet, K., Saenphet, S., and Thitaram, C. (2016). Uvaria rufa Blume attenuates benign prostatic hyperplasia via inhibiting $5 \alpha-$ reductase and enhancing antioxidant status. J. Ethnopharmacol. 194, 483-494. doi: 10.1016/j.jep.2016.10.036

Caparros-Lefebvre, D., and Lees, A. J. (2005). Atypical unclassifiable parkinsonism on Guadeloupe: an environmental toxic hypothesis. Mov. Disord. 20, S114S118. doi: $10.1002 / \mathrm{mds} .20553$

Casteilla, L., Rigoulet, M., and Pénicaud, L. (2001). Mitochondrial ROS metabolism: modulation by uncoupling proteins. IUBMB Life 52, 181-188. doi: $10.1080 / 15216540152845984$

Cercato, L. M., White, P. A., Nampo, F. K., Santos, M. R., and Camargo, E. A. (2015). A systematic review of medicinal plants used for weight loss in Brazil: is there potential for obesity treatment? J. Ethnopharmacol. 176, 286-296. doi: 10.1016/j.jep.2015.10.038

Chadha, Y. (1985). The Wealth of India. Raw Materials, Vol. I. Minneapolis, MN: Publications and Information Directorate; Council of Scientific and Industrial Research.

Champy, P., Höglinger, G. U., Féger, J., Gleye, C., Hocquemiller, R., Laurens, A., et al. (2004). Annonacin, a lipophilic inhibitor of mitochondrial complex I, induces nigral and striatal neurodegeneration in rats: possible relevance for atypical parkinsonism in Guadeloupe. J. Neurochem. 88, 63-69. doi: 10.1046/j.1471-4159.2003.02138.x

Chan, H. H., Hwang, T. L., Thang, T. D., Leu, Y. L., Kuo, P. C., Nguyet, B. T., et al. (2013). Isolation and synthesis of melodamide A, a new anti-inflammatory phenolic amide from the leaves of Melodorum fruticosum. Planta Med. 79, 288-294. doi: 10.1055/s-0032-1328131

Chang, F. R., Hwang, T. L., Yang, Y. L., Li, C. E., Wu, C. C., Issa, H. H., et al. (2006). Anti-inflammatory and cytotoxic diterpenes from formosan Polyalthia longifolia var. pendula. Planta Med 72, 1344-1347. doi: 10.1055/s-2006-951691

Chang, F. R., Wei, J. L., Teng, C. M., and Wu, Y. C. (1998). Two new 7-dehydroaporphine alkaloids and antiplatelet action aporphines from the leaves of Annona purpurea. Phytochemistry 49, 2015-2018. doi: 10.1016/S0031-9422(98)00376-8 
Chang, F. R., and Wu, Y. C. (2001). Novel cytotoxic annonaceous acetogenins from Annona muricata. J. Nat. Prod. 64, 925-931. doi: 10.1021/np010035s

Chang, H. L., Chang, F. R., Chen, J. S., Wang, H. P., Wu, Y. H., Wang, C. C., et al. (2008). Inhibitory effects of 16-hydroxycleroda-3,13(14)Edien-15-oic acid on superoxide anion and elastase release in human neutrophils through multiple mechanisms. Eur. J. Pharmacol. 586, 332-339. doi: 10.1016/j.ejphar.2008.02.041

Chavan, M. J., Kolhe, D. R., Wakte, P. S., and Shinde, D. B. (2012). Analgesic and antiinflammatory activity of kaur-16-en-19-oic acid from Annona reticulata L. bark. Phytother. Res. 26, 273-276. doi: 10.1002/ptr.3544

Chavan, M. J., Wakte, P. S., and Shinde, D. B. (2010). Analgesic and antiinflammatory activity of Caryophyllene oxide from Annona squamosa L. bark. Phytomedicine 17, 149-151. doi: 10.1016/j.phymed.2009.05.016

Chen, J., Pan, W., Yu, H., Huang, S., and Zhu, P. (2016). GW27-e1067 Resveratrol protects against TNF-[alpha]-induced injury in human umbilical endothelial cells through promoting sirtuin-1-induced repression of NF-KB and p38 MAPK. J. Am. Coll. Cardiol. 68, C38. doi: 10.1016/j.jacc.2016.07.141

Chen, Y., Chen, J. W., Wang, Y., Xu, S. S., and Li, X. (2012). Six cytotoxic annonaceous acetogenins from Annona squamosa seeds. Food Chem. 135, 960-966. doi: 10.1016/j.foodchem.2012.05.041

Chen, Y., and Junger, W. G. (2012). Measurement of oxidative burst in neutrophils. Methods Mol. Biol. 844, 115-124. doi: 10.1007/978-1-61779-527-5_8

Cheng, X., Xiao, Y., Wang, X., Wang, P., Li, H., Yan, H., et al. (2012). Antitumor and pro-apoptotic activity of ethanolic extract and its various fractions from Polytrichum commune L.ex Hedw in L1210 cells. J. Ethnopharmacol. 143, 49-56. doi: 10.1016/j.jep.2012.05.054

Chokchaisiri, R., Chaichompoo, W., Chalermglin, R., and Suksamrarn, A. (2015). Potent antiplasmodial alkaloids and flavonoids from Dasymaschalon acuminatum. Rec. Nat. Prod. 9, 243.

Chuang, P. H., Hsieh, P. W., Yang, Y. L., Hua, K. F., Chang, F. R., Shiea, J., et al. (2008). Cyclopeptides with anti-inflammatory activity from seeds of Annona montana. J. Nat. Prod. 71, 1365-1370. doi: 10.1021/np8001282

Chun, K. S., and Surh, Y. J. (2004). Signal transduction pathways regulating cyclooxygenase-2 expression: potential molecular targets for chemoprevention. Biochem. Pharmacol. 68, 1089-1100. doi: 10.1016/j.bcp.2004.05.031

Cirino, G., Fiorucci, S., and Sessa, W. C. (2003). Endothelial nitric oxide synthase: the Cinderella of inflammation? Trends Pharmacol. Sci. 24, 91-95. doi: 10.1016/S0165-6147(02)00049-4

Cominelli, F. (2004). Cytokine-based therapies for Crohn's disease-new paradigms. New Engl. J. Med. 351, 2045-2048. doi: 10.1056/NEJMp048253

Cooke, J. P., and Dzau, V. J. (1997). Derangements of the nitric oxide synthase pathway, L-arginine, and cardiovascular diseases. Circulation 96, 379-382.

Couvreur, T. L., Pirie, M. D., Chatrou, L. W., Saunders, R. M., Su, Y. C., Richardson, J. E., et al. (2011). Early evolutionary history of the flowering plant family Annonaceae: steady diversification and boreotropical geodispersal. J. Biogeogr. 38, 664-680. doi: 10.1111/j.1365-2699.2010.02434.x

Dabrowski, A., Konturek, S. J., Konturek, J. W., and Gabryelewicz, A. (1999). Role of oxidative stress in the pathogenesis of caerulein-induced acute pancreatitis. Eur. J. Pharmacol. 377, 1-11. doi: 10.1016/S0014-2999(99)00421-5

Dannenberg, A. J., Altorki, N. K., Boyle, J. O., Dang, C., Howe, L. R., Weksler, B. B., et al. (2001). Cyclo-oxygenase 2: a pharmacological target for the prevention of cancer. Lancet Oncol. 2, 544-551. doi: 10.1016/S1470-2045(01)00488-0

Davis, C. C., and Wurdack, K. J. (2004). Host-to-parasite gene transfer in flowering plants: phylogenetic evidence from Malpighiales. Science 305, 676-678. doi: 10.1126/science.1100671

de Albuquerque Montenegro, C., de Morais Lima, G. R., Gomes, I. F., Tavares, J. F., and Batista, L. M. (2014). Gastroprotective effect of Xylopia langsdorffiana A. St.-Hil. \& Tul.(Annonaceae): involvement of endogenous sulfhydryls compounds and nitric oxide. Records Nat. Prod. 8, 165.

De Fátima, A., Modolo, L. V., Conegero, L. S., Pilli, R. A., Ferreira, C. V., Kohn, L. K., et al. (2006). Styryl lactones and their derivatives: biological activities, mechanisms of action and potential leads for drug design. Curr. Med. Chem. 13, 3371-3384. doi: 10.2174/092986706779010298

DeFilipps, R. A., Maina, S. L., and Crepin, J. (2004). Medicinal Plants of the Guianas (Guyana, Surinam, French Guiana). Washington, DC: Department of Botany, National Museum of Natural History, Smithsonian Institution.

Dellai, A., Maricic, I., Kumar, V., Arutyunyan, S., Bouraoui, A., and Nefzi, A. (2010). Parallel synthesis and anti-inflammatory activity of cyclic peptides cyclosquamosin D and Met-cherimolacyclopeptide B and their analogs. Bioorg. Med. Chem. Lett. 20, 5653-5657. doi: 10.1016/j.bmcl.2010.08.033

DelloStritto, D. J., Connell, P. J., Dick, G. M., Fancher, I. S., Klarich, B., Fahmy, J. N., et al. (2016). Differential regulation of TRPV1 channels by H2O2: implications for diabetic microvascular dysfunction. Basic Res. Cardiol. 111, 1-16. doi: 10.1007/s00395-016-0539-4

De Simone, V., Franze, E., Ronchetti, G., Colantoni, A., Fantini, M., Di Fusco, D., et al. (2015). Th17-type cytokines, IL-6 and TNF- $\alpha$ synergistically activate STAT3 and NF-kB to promote colorectal cancer cell growth. Oncogene 34, 3493-3503. doi: 10.1038/onc.2014.286

de Sousa, O. V., Vieira, G. D., de Jesus, R. G., Yamamoto, C. H., and Alves, M. S. (2010). Antinociceptive and anti-inflammatory activities of the ethanol extract of Annona muricata L. leaves in animal models. Int. J. Mol. Sci. 11, 2067-2078. doi: $10.3390 /$ ijms 11052067

Dey, R., Majumder, N., Bhattacharyya Majumdar, S., Bhattacharjee, S., Banerjee, S., Roy, S., et al. (2007). Induction of host protective Th1 immune response by chemokines in Leishmania donovani-infected BALB/c mice. Scand. J. Immunol. 66, 671-683. doi: 10.1111/j.1365-3083.2007.02025.x

Diels, O., and Alder, K. (1932). Synthesen in der hydroaromatischen Reihe. XVII. Mitteilung.("Dien-Synthesen" $\mathrm{n}$ in der hydroaromatischen Reihe. XVII. MSynthesen des Pyridins, Chinolins, Chinaldins und Isochinolins.). Justus Liebigs Annalen Chem. 498, 16-49. doi: 10.1002/jlac.19324980103

Dinarello, C. (1997). Role of pro-and anti-inflammatory cytokines during inflammation: experimental and clinical findings. J. Biol. Regul. Homeost. Agents 11, 91-103.

Drake, I. M., Mapstone, N. P., Schorah, C. J., White, K. L., Chalmers, D. M., Dixon, M. F., et al. (1998). Reactive oxygen species activity and lipid peroxidation in Helicobacter pylori associated gastritis: relation to gastric mucosal ascorbic acid concentrations and effect of $H$. pylori eradication. Gut 42, 768-771. doi: $10.1136 /$ gut.42.6.768

Duke, J. (2000). Duke's Phytochemical and Ethnobotanical Databases, Phytochemical Database. Beltsville, MD: USDAARS-NGRL, Beltsville Agricultural Research Centre.

Duke, J., and Beckstrom-Sternberg, S. (2000). Phytochemical Database USDAARS-NGRL. Beltsbille Agricultural Research Center. Beltsbille, MD. Available online at: http://www.ars.grin.gov/cgl-bin/duke/ethnobot.pl (Accessed August $23,2011)$.

Dunal, M. F. (1817). Monographie de la Famille des Anonacées. chez Treuttel et Würtz.

Duque, J., Fresno, M., and Iñiguez, M. A. (2005). Expression and function of the nuclear factor of activated $\mathrm{T}$ cells in colon carcinoma cells involvement in the regulation of cyclooxygenase-2. J. Biol. Chem. 280, 8686-8693. doi: 10.1074/jbc.M413076200

Duraipandiyan, V., Ayyanar, M., and Ignacimuthu, S. (2006). Antimicrobial activity of some ethnomedicinal plants used by Paliyar tribe from Tamil Nadu, India. BMC Complement. Altern. Med. 6:35. doi: 10.1186/1472-6882-6-35

Esquenazi, S., and Bazan, H. E. (2010). Role of platelet-activating factor in cell death signaling in the cornea: a review. Mol. Neurobiol. 42, 32-38. doi: 10.1007/s12035-010-8129-1

Faria Lua Figueiredo, S., Regina Campos Viana, V., Simões, C., Albarello, N., Carlos Trugo, L., Auxiliadora Coelho Kaplan, M., et al. (1999). Lignans from leaves, seedlings and micropropagated plants of Rollinia mucosa (Jacq.) Baill.-Annonaceae. Plant Cell Tissue Organ Cult. 56, 121-124. doi: 10.1023/A:1006258121633

Foong, C. P., and Hamid, R. A. (2012). Evaluation of anti-inflammatory activities of ethanolic extract of Annona muricata leaves. Rev. Bras. Farmacog. 22, 1301-1307. doi: 10.1590/S0102-695X2012005000096

Formagio, A. S., Kassuya, C. A., Neto, F. F., Volobuff, C. R., Iriguchi, E. K., Vieira Mdo, C., et al. (2013a). The flavonoid content and antiproliferative, hypoglycaemic, anti-inflammatory and free radical scavenging activities of Annona dioica St. Hill. BMC Complement. Altern. Med. 13:14. doi: 10.1186/1472-6882-13-14

Formagio, A. S., Vieira Mdo, C., Dos Santos, L. A., Cardoso, C. A., Foglio, M. A., de Carvalho, J. E., et al. (2013b). Composition and evaluation of the anti-inflammatory and anticancer activities of the essential oil from Annona sylvatica A. St.-Hil. J. Med. Food 16, 20-25. doi: 10.1089/jmf.2011.0303

Frausin, G., Lima, R. B. S., Hidalgo, A. F., Maas, P., and Pohlit, A. M. (2014). Plants of the Annonaceae traditionally used as antimalarials: a 
review. Rev. Bras. Frutic. 36, 315-337. doi: 10.1590/S0100-294520140005 00038

Fries, J. H. (1959). Factors influencing clinical evaluation of food allergy. Pediatr. Clin. North Am. 6, 867-880. doi: 10.1016/S0031-3955(16)30839-2

Garavito, G., Rincón, J., Arteaga, L., Hata, Y., Bourdy, G., Gimenez, A., et al. (2006). Antimalarial activity of some Colombian medicinal plants. J. Ethnopharmacol. 107, 460-462. doi: 10.1016/j.jep.2006.03.033

Ge, Y. W., Zhu, S., Shang, M. Y., Zang, X. Y., Wang, X., Bai, Y. J., et al. (2013). Aristololactams and aporphines from the stems of Fissistigma oldhamii (Annonaceae). Phytochemistry 86, 201-207. doi: 10.1016/j.phytochem.2012.09.011

Gebicke-Haerter, P. J. (2001). Microglia in neurodegeneration: molecular aspects. Microsc. Res. Technol. 54, 47-58. doi: 10.1002/jemt.1120

Ghosh, A., Das, B. K., Chatterjee, S. K., and Chandra, G. (2008). Antibacterial potentiality and phytochemical analysis of mature leaves of Polyalthia longifolia (Magnoliales: Annonaceae). South Pac. J. Nat. Sci. 26, 68-72. doi: 10.1071/SP08011

González-Esquinca, A. R., De-La-Cruz-Chacón, I., Castro-Moreno, M., OrozcoCastillo, J. A., and Riley-Salda-a, C. A. (2014). Alkaloids and acetogenins in Annonaceae development: biological considerations. Rev. Bras. Frutic. 36, 01-16. doi: 10.1590/S0100-29452014000500001

Gordon, S., and Taylor, P. R. (2005). Monocyte and macrophage heterogeneity. Nat. Rev. Immunol. 5, 953-964. doi: 10.1038/nri1733

Greten, F. R., Eckmann, L., Greten, T. F., Park, J. M., Li, Z. W., Egan, L. J., et al. (2004). IKK $\beta$ links inflammation and tumorigenesis in a mouse model of colitis-associated cancer. Cell 118, 285-296. doi: 10.1016/j.cell.2004.07.013

Grivennikov, S. I., Greten, F. R., and Karin, M. (2010). Immunity, inflammation, and cancer. Cell 140, 883-899. doi: 10.1016/j.cell.2010.01.025

Guo, H., Callaway, J. B., and Ting, J. P. (2015). Inflammasomes: mechanism of action, role in disease, and therapeutics. Nat. Med. 21, 677. doi: $10.1038 / \mathrm{nm} .3893$

Halliwell, B. (2006). Reactive species and antioxidants. Redox biology is a fundamental theme of aerobic life. Plant Physiol. 141, 312-322. doi: $10.1104 /$ pp.106.077073

Hamonnière, M., Leboeuf, M., and Cavé, A. (1977). Alcaloides aporphiniques et composés terpéniques du Polyalthia oliveri. Phytochemistry 16, 1029-1034. doi: 10.1016/S0031-9422(00)86716-3

Han, B., Wang, T. D., Shen, S. M., Yu, Y., Mao, C., Yao, Z. J., et al. (2015). Annonaceous acetogenin mimic AA005 induces cancer cell death via apoptosis inducing factor through a caspase-3-independent mechanism. BMC Cancer 15:139. doi: 10.1186/s12885-015-1133-0

Harrington, L. S., Lucas, R., McMaster, S. K., Moreno, L., Scadding, G., Warner, T. D., et al. (2008). COX-1, and not COX-2 activity, regulates airway function: relevance to aspirin-sensitive asthma. FASEB J. 22, 4005-4010. doi: 10.1096/fj.08-107979

Hoes, J. N., Jacobs, J. W., Buttgereit, F., and Bijlsma, J. W. (2010). Current view of glucocorticoid co-therapy with DMARDs in rheumatoid arthritis. Nat. Rev. Rheumatol. 6, 693-702. doi: 10.1038/nrrheum.2010.179

Hsu, Y. M., Wu, T. Y., Du, Y. C., El-Shazly, M., Beerhues, L., Thang, T. D., et al. (2016). 3-Methyl-4,5-dihydro-oxepine, polyoxygenated seco-cyclohexenes and cyclohexenes from Uvaria flexuosa and their anti-inflammatory activity. Phytochemistry 122, 184-192. doi: 10.1016/j.phytochem.2015.12.013

Hu, X. D., Yang, Y., Zhong, X. G., Zhang, X. H., Zhang, Y. N., Zheng, Z. P., et al. (2008). Anti-inflammatory effects of Z23 on LPS-induced inflammatory responses in RAW264.7 macrophages. J. Ethnopharmacol. 120, 447-451. doi: 10.1016/j.jep.2008.09.026

Huscher, D., Thiele, K., Gromnica-Ihle, E., Hein, G., Demary, W., Dreher, R., et al. (2009). Dose-related patterns of glucocorticoid-induced side effects. Ann. Rheum. Dis. 68, 1119-1124. doi: 10.1136/ard.2008.092163

Hu, X.-D., Zhong, X.-G., Zhang, X.-H., Zhang, Y.-N., Zheng, Z.-P., Zhou, Y., et al. (2007). $7^{\prime}$-(3', 4'-dihydroxyphenyl)-N-[(4-methoxyphenyl)ethyl]propenamide (Z23), an effective compound from the Chinese herb medicine Fissistigma oldhamii (Hemsl.) Merr, suppresses T cell-mediated immunity in vitro and in vivo. Life Sci. 81, 1677-1684. doi: 10.1016/j.lfs.2007.10.004

Hutchinson, J. (1959). The Families of Flowering Plants, Vol. II. Monocotyledons, 2nd Edn. Clarendon Press; Oxford University Press.

Hwang, T. L., Li, G. L., Lan, Y. H., Chia, Y. C., Hsieh, P. W., Wu, Y. H., et al. (2009). Potent inhibition of superoxide anion production in activated human neutrophils by isopedicin, a bioactive component of the Chinese medicinal herb Fissistigma oldhamii. Free Radic. Biol. Med. 46, 520-528. doi: 10.1016/j.freeradbiomed.2008.11.014

Ishola, I. O., Awodele, O., Olusayero, A. M., and Ochieng, C. O. (2014). Mechanisms of analgesic and anti-inflammatory properties of Annona muricata Linn. (Annonaceae) fruit extract in rodents. J. Med. Food 17, 1375-1382. doi: 10.1089/jmf.2013.0088

Ishola, I. O., Ikumawoyi, V. O., Afolayan, G. O., and Olorife, O. J. (2016). Antinociceptive and anti-inflammatory properties of hydroethanolic seed extract of Monodora myristica (Annonaceae) in rodents. West Afr. J. Pharm. $27,22-32$.

Iñiguez, M. A., Martinez-Martinez, S., Punzón, C., Redondo, J. M., and Fresno, M. (2000). An essential role of the nuclear factor of activated $\mathrm{T}$ cells in the regulation of the expression of the cyclooxygenase- 2 gene in human $\mathrm{T}$ lymphocytes. J. Biol. Chem. 275, 23627-23635. doi: 10.1074/jbc.M001381200

Jachak, S. M. (2006). Cyclooxygenase inhibitory natural products: current status. Curr. Med. Chem. 13, 659-678. doi: 10.2174/092986706776055698

Jantan, I., Rafi, I. A., and Jalil, J. (2005). Platelet-activating factor (PAF) receptorbinding antagonist activity of Malaysian medicinal plants. Phytomedicine 12, 88-92. doi: 10.1016/j.phymed.2003.06.006

Jiménez, V. M., Gruschwitz, M., Schweiggert, R. M., Carle, R., and Esquivel, P. (2014). Identification of phenolic compounds in soursop (Annona muricata) pulp by high-performance liquid chromatography with diode array and electrospray ionization mass spectrometric detection. Food Res. Int. 65, 42-46. doi: 10.1016/j.foodres.2014.05.051

Johnson, T. A., Sohn, J., Ward, A. E., Cohen, T. L., Lorig-Roach, N. D., Chen, H., et al. (2013). (+)-Altholactone exhibits broad spectrum immune modulating activity by inhibiting the activation of pro-inflammatory cytokines in RAW 264.7 cell lines. Bioorg. Med. Chem. 21, 4358-4364. doi: 10.1016/j.bmc.2013.04.055

Kandimalla, R., Dash, S., Kalita, S., Choudhury, B., Malampati, S., Devi, R., et al. (2017). Bioactive fraction of Annona reticulata bark (or) Ziziphus jujuba root bark along with insulin attenuates painful diabetic neuropathy through inhibiting NF-кB inflammatory cascade. Front. Cell. Neurosci. 11:73. doi: $10.3389 /$ fncel.2017.00073

Kandimalla, R., Dash, S., Kalita, S., Choudhury, B., Malampati, S., Kalita, K., et al. (2016). Bioactive guided fractions of Annona reticulata L. bark: protection against liver toxicity and inflammation through inhibiting oxidative stress and proinflammatory cytokines. Front. Pharmacol. 7:168. doi: 10.3389/fphar.2016.00168

Karin, M., and Ben-Neriah, Y. (2000). Phosphorylation meets ubiquitination: the control of NF-кB activity. Annu. Rev. Immunol. 18, 621-663. doi: 10.1146/annurev.immunol.18.1.621

Karin, M., Lawrence, T., and Nizet, V. (2006). Innate immunity gone awry: linking microbial infections to chronic inflammation and cancer. Cell 124, 823-835. doi: 10.1016/j.cell.2006.02.016

Kasperska-Zajac, A., Brzoza, Z., and Rogala, B. (2008). Platelet-activating factor (PAF): a review of its role in asthma and clinical efficacy of PAF antagonists in the disease therapy. Recent Pat. Inflamm. Allergy Drug Discov. 2, 72-76. doi: 10.2174/187221308783399306

Katkar, K. V., Suthar, A. C., and Chauhan, V. S. (2010). The chemistry, pharmacologic, and therapeutic applications of Polyalthia longifolia. Pharmacogn. Rev. 4, 62-68. doi: 10.4103/0973-7847.65329

Kidd, B. L., and Urban, L. A., (2001). Mechanisms of inflammatory pain. Br. J. Anaesth. 87, 3-11. doi: 10.1093/bja/87.1.3

Kim, B. W., Koppula, S., Kumar, H., Park, J. Y., Kim, I. W., More, S. V., et al. (2015). alpha-Asarone attenuates microglia-mediated neuroinflammation by inhibiting NF kappa B activation and mitigates MPTP-induced behavioral deficits in a mouse model of Parkinson's disease. Neuropharmacology 97, 46-57. doi: 10.1016/j.neuropharm.2015.04.037

Koek-Noorman, J., and Westra, L. Y. (2012). Macrophotographic wood atlas of Annonaceae. Bot. J. Linnean Soc. 169, 135-189. doi: 10.1111/j.1095-8339.2012.01237.x

Kojima, N., and Tanaka, T. (2009). Medicinal chemistry of Annonaceous acetogenins: design, synthesis, and biological evaluation of novel analogues. Molecules 14, 3621-3661. doi: 10.3390/molecules14093621

Kouam, S. F., Ngouonpe, A. W., Lamshöft, M., Talontsi, F. M., Bauer, J. O., Strohmann, C., et al. (2014). Indolosesquiterpene alkaloids from the 
Cameroonian medicinal plant Polyalthia oliveri (Annonaceae). Phytochemistry 105, 52-59. doi: 10.1016/j.phytochem.2014.06.015

Kuate, D., Etoundi, B. C. O., Soukontoua, Y. B., Ngondi, J. L., and Oben, J. E. (2011). Comparative study of the antioxidant, free radical scavenging activity and human LDL oxidation inhibition of three extracts from seeds of a Cameroonian spice, Xylopia parviflora (A. Rich.) Benth. (Annonaceae). Int. J. Biomed. Pharm. Sci 5, 18-30.

Kumar, G. P., and Khanum, F. (2012). Neuroprotective potential of phytochemicals. Pharmacogn. Rev. 6, 81. doi: 10.4103/0973-7847.99898

Kuo, K. K., Chen, Y. L., Chen, L. R., Li, C. F., Lan, Y. H., Chang, F. R., et al. (2011). Involvement of phorbol-12-myristate-13-acetate-induced protein 1 in goniothalamin-induced TP53-dependent and-independent apoptosis in hepatocellular carcinoma-derived cells. Toxicol. Appl. Pharmacol. 256, 8-23. doi: $10.1016 / j$.taap.2011.07.002

Kuo, R. Y., Chang, F. R., Chen, C. Y., Teng, C. M., Yen, H. F., and Wu, Y. C. (2001). Antiplatelet activity of N-methoxycarbonyl aporphines from Rollinia mucosa. Phytochemistry 57, 421-425. doi: 10.1016/S0031-9422(01)00076-0

Lage, G. A., Medeiros, Fda. S., Furtado, Wde. L., Takahashi, J. A., de Souza Filho, J. D., and Pimenta, L. P. (2014). The first report on flavonoid isolation from Annona crassiflora Mart. Nat. Prod. Res. 28, 808-811. doi: 10.1080/14786419.2014.885518

Lannuzel, A., Michel, P. P., Caparros-Lefebvre, D., Abaul, J., Hocquemiller, R., and Ruberg, M. (2002). Toxicity of Annonaceae for dopaminergic neurons: potential role in atypical parkinsonism in Guadeloupe. Mov. Disord. 17, 84-90. doi: $10.1002 / \mathrm{mds} .1246$

Lannuzel, A., Michel, P. P., Höglinger, G. U., Champy, P., Jousset, A., Medja, F., et al. (2003). The mitochondrial complex i inhibitor annonacin is toxic to mesencephalic dopaminergic neurons by impairment of energy metabolism. Neuroscience 121, 287-296. doi: 10.1016/S0306-4522(03)00441-X

Liaw, C. C., Wu, T. Y., Chang, F. R., and Wu, Y. C. (2010). Historic perspectives on Annonaceous acetogenins from the chemical bench to preclinical trials. Planta Med. 76, 1390-1404. doi: 10.1055/s-0030-1250006

Liou, J. R., Wu, T. Y., Thang, T. D., Hwang, T. L., Wu, C. C., Cheng, Y. B., et al. (2014). Bioactive 6S-styryllactone constituents of Polyalthia parviflora. J. Nat. Prod. 77, 2626-2632. doi: $10.1021 / \mathrm{np5004577}$

López, M. L., Hernández, A., Chamorro, G., and Mendoza-Figueroa, T. (1993). aAsarone toxicity in long-term cultures of adult rat hepatocytes. Planta Med. 59, 115-120. doi: 10.1055/s-2006-959624

López, R., Cuca, L. E., and Delgado, G. (2009). Antileishmanial and immunomodulatory activity of Xylopia discreta. Parasite Immunol. 31, 623-630. doi: 10.1111/j.1365-3024.2009.01134.x

Ludolph, A. C., Kassubek, J., Landwehrmeyer, B. G., Mandelkow, E., Mandelkow, E. M., Burn, D. J., et al. (2009). Tauopathies with parkinsonism: clinical spectrum, neuropathologic basis, biological markers, and treatment options. Eur. J. Neurol. 16, 297-309. doi: 10.1111/j.1468-1331.2008.02513.x

Malebo, H. M., Wenzler, T., Cal, M., Swaleh, S. M., Omolo, M. O., Hassanali, A., et al. (2013). Anti-protozoal activity of aporphine and protoberberine alkaloids from Annickia kummeriae (Engl. \& Diels) Setten \& Maas (Annonaceae). BMC Complement. Altern. Med. 13:48. doi: 10.1186/1472-6882-13-48

Mantovani, A., Allavena, P., Sica, A., and Balkwill, F. (2008). Cancer-related inflammation. Nature 454, 436-444. doi: 10.1038/nature07205

Mantovani, A., Garlanda, C., and Allavena, P. (2010). Molecular pathways and targets in cancer-related inflammation. Ann. Med. 42, 161-170. doi: $10.3109 / 07853890903405753$

Marok, R., Winyard, P. G., Coumbe, A., Kus, M. L., Gaffney, K., Blades, S., et al. (1996). Activation of the transcription factor nuclear factorkappaB in human inflamed synovial tissue. Arthritis Rheum. 39, 583-591. doi: $10.1002 /$ art.1780390407

McGeer, P. L., and McGeer, E. G. (2004). Inflammation and neurodegeneration in Parkinson's disease. Parkinson Relat. Disord. 10, S3-S7. doi: 10.1016/j.parkreldis.2004.01.005

Meddah, B., Mamadou, G., Tiendrebeogo, R., Limas-Nzouzi, N., Cherrah, Y., Eto, B., et al. (2013). Analgesic, anti-inflammatory and antidepressant activities of triterpene frommeiocarpidium lepidotum (annonaceae) bark. Fundam. Clin. Pharmacol. 27, 118-119.

Meira, C. S., Guimarães, E. T., Macedo, T. S., da Silva, T. B., Menezes, L. R., Costa, E. V., et al. (2015). Chemical composition of essential oils from Annona vepretorum Mart. and Annona squamosa L.(Annonaceae) leaves and their antimalarial and trypanocidal activities. J. Essent. Oil Res. 27, 160-168. doi: 10.1080/10412905.2014.982876

Mittal, M., Siddiqui, M. R., Tran, K., Reddy, S. P., and Malik, A. B. (2014). Reactive oxygen species in inflammation and tissue injury. Antioxid. Redox Signal. 20, 1126-1167. doi: 10.1089/ars.2012.5149

Moghadamtousi, S. Z., Fadaeinasab, M., Nikzad, S., Mohan, G., Ali, H. M., and Kadir, H. A. (2015). Annona muricata (Annonaceae): a review of its traditional uses, isolated acetogenins and biological activities. Int. J. Mol. Sci. 16, 15625-15658. doi: 10.3390/ijms160715625

Moghadamtousi, S. Z., Goh, B. H., Chan, C. K., Shabab, T., and Kadir, H. A. (2013). Biological activities and phytochemicals of Swietenia macrophylla King. Molecules 18, 10465-10483. doi: 10.3390/molecules 180910465

Moghadamtousi, S. Z., Kadir, H. A., Paydar, M., Rouhollahi, E., and Karimian, H. (2014a). Annona muricata leaves induced apoptosis in A549 cells through mitochondrial-mediated pathway and involvement of NF-кB. BMC Complement. Altern. Med. 14:299. doi: 10.1186/1472-6882-14-299

Moghadamtousi, S. Z., Rouhollahi, E., Karimian, H., Fadaeinasab, M., Abdulla, M. A., and Kadir, H. A. (2014b). Gastroprotective activity of Annona muricata leaves against ethanol-induced gastric injury in rats via Hsp70/Bax involvement. Drug Des. Devel. Ther. 8, 2099-2110. doi: 10.2147/DDDT.S70096

Moharam, B. A., Jantan, I., Ahmad, F. B., and Jalil, J. (2010a). Antiplatelet aggregation and platelet activating factor (PAF) receptor antagonistic activities of the essential oils of five Goniothalamus species. Molecules 15, 5124-5138. doi: 10.3390/molecules 15085124

Moharam, B. A., Jantan, I., Jalil, J., and Ahmad, F. (2012). Inhibitory effect of compounds from Goniothalamus tapis Miq. and Goniothalamus uvaroides King on platelet-activating factor receptor binding. Phytother. Res. 26, 687-691. doi: $10.1002 /$ ptr.3620

Moharam, B. A., Jantan, I., Jalil, J., and Shaari, K. (2010b). Inhibitory effects of phylligenin and quebrachitol isolated from Mitrephora vulpina on platelet activating factor receptor binding and platelet aggregation. Molecules 15, 7840-7848. doi: 10.3390/molecules 15117840

Moncada, S., Gryglewski, R. J., Bunting, S., and Vane, J. R. (1976). A lipid peroxide inhibits the enzyme in blood vessel microsomes that generates from prostaglandin endoperoxides the substance (prostaglandin $\mathrm{X})$ which prevents platelet aggregation. Prostaglandins 12, 715-737. doi: 10.1016/0090-6980(76)90048-4

Moody, J. O., Ogundipe, O. D., Akang, E., and Agbedana, E. (2007). Toxicological studies on the purified protoberberine alkaloidal fraction of Enantia chlorantha Oliv (ANNONACEAE). Afr. J. Med. Med. Sci. 36, 317-323.

Moreira, I. C., Roque, N. F., Vilegas, W., Zalewski, C. A., Lago, J. H., and Funasaki, M. (2013). Genus Xylopia (Annonaceae): chemical and biological aspects. Chem. Biodivers. 10, 1921-1943. doi: 10.1002/cbdv.201100308

Moukette, B. M., Pieme, C. A., Njimou, J. R., Biapa, C. P., Marco, B., and Ngogang, J. Y. (2015). In vitro antioxidant properties, free radicals scavenging activities of extracts and polyphenol composition of a non-timber forest product used as spice: Monodora myristica. Biol. Res. 48, 15. doi: 10.1186/s40659-0150003-1

Nakahira, K., Haspel, J. A., Rathinam, V. A., Lee, S. J., Dolinay, T., Lam, H. C., et al. (2011). Autophagy proteins regulate innate immune responses by inhibiting the release of mitochondrial DNA mediated by the NALP3 inflammasome. Nat. Immunol. 12, 222-230. doi: 10.1038/ni.1980

Nguyen, T. H., Ho, V. D., Do, T. T., Bui, H. T., Phan, V. K., Sak, K., et al. (2015). A new lignan glycoside from the aerial parts and cytotoxic investigation of Uvaria rufa. Nat. Prod. Res. 29, 247-252. doi: 10.1080/14786419.2014.971790

Nhiem, N. X., Hien, N. T., Tai, B. H., Anh, H. L., Hang, D. T., Quang, T. H., et al. (2015). New ent-kauranes from the fruits of Annona glabra and their inhibitory nitric oxide production in LPS-stimulated RAW264. 7 macrophages. Bioorgan. Med. Chem. Lett. 25, 254-258. doi: 10.1016/j.bmcl.2014.11.059

Nishiyama, Y., Moriyasu, M., Ichimaru, M., Iwasa, K., Kato, A., Mathenge, S. G., et al. (2006). Secondary and tertiary isoquinoline alkaloids from Xylopia parviflora. Phytochemistry 67, 2671-2675. doi: 10.1016/j.phytochem.2006.07.011

Njoku, U. O. (2007). Antioxidant Activity of Seed Extract and Fractions of Monodora Tenuifolia (Enugu: Nigerian University Library Press).

Nordin, N., Jalil, J., Jantan, I., and Murad, S. (2012). Platelet-activating factor (PAF) receptor binding activity of the roots of Enicosanthellum pulchrum. Pharm. Biol. 50, 284-290. doi: 10.3109/13880209.2011.602416 
Nordin, N., Salama, S. M., Golbabapour, S., Hajrezaie, M., Hassandarvish, P., Kamalidehghan, B., et al. (2014). Anti-ulcerogenic effect of methanolic extracts from Enicosanthellum pulchrum (King) Heusden against ethanolinduced acute gastric lesion in animal models. PLOS ONE 9:e111925. doi: 10.1371/journal.pone.0111925

Obiri, D. D., and Osafo, N. (2013). Aqueous ethanol extract of the fruit of Xylopia aethiopica (Annonaceae) exhibits anti-anaphylactic and anti-inflammatory actions in mice. J. Ethnopharmacol. 148, 940-945. doi: $10.1016 /$ j.jep.2013.05.047

Odalo, J. O., Joseph, C. C., Nkunya, M. H., Sattler, I., Lange, C., Friedrich, G., et al. (2010). Aristolactams, 1-(2-C-methyl-beta-D-ribofuranosyl)-uracil and other bioactive constituents of Toussaintia orientalis. Nat. Prod. Commun. 5, 253-258.

Odukoya, O. A., Ilori, O. O., Sofidiya, M. O., Aniunoh, O. A., Lawal, B. M., and Tade, I. O. (2005). Antioxidant activity of Nigerian dietary spices. Elect. J. Environ. Agric. Food Chem. 4, 1086-1093.

Ofukwu, R., Ayoola, A., and Akwuobu, C. (2008). Medicinal plants used in treatment of tuberculosis in humans and animals by idoma tribe of North Central Nigeria. Nigerian Veterin. J. 29, 25-30.

Ogbonnia, S., Adekunle, A., Bosa, M., and Enwuru, V. (2008). Evaluation of acute and subacute toxicity of Alstonia congensis Engler (Apocynaceae) bark and Xylopia aethiopica (Dunal) A. Rich (Annonaceae) fruits mixtures used in the treatment of diabetes. Afr. J. Biotechnol. 7, 701-705.

Ogunkunle, A., and Ladejobi, T. A. (2006). Ethnobotanical and phytochemical studies on some species of Senna in Nigeria. Afr. J. Biotechnol. 5, 2020-2023.

Olefsky, J. M., and Glass, C. K. (2010). Macrophages, inflammation, and insulin resistance. Annu. Rev. Physiol. 72, 219-246. doi: 10.1146/annurev-physiol-021909-135846

Opferman, J. T. (2008). Apoptosis in the development of the immune system. Cell Death Differ. 15, 234-242. doi: 10.1038/sj.cdd.4402182

Orlikova, B., Schumacher, M., Juncker, T., Yan, C. C., Inayat-Hussain, S. H., Hajjouli, S., et al. (2013). Styryl-lactone goniothalamin inhibits TNFalpha-induced NF-kappaB activation. Food Chem. Toxicol. 59, 572-578. doi: 10.1016/j.fct.2013.06.051

Parmar, V. S., Tyagi, O. D., Malhotra, A., Singh, S. K., Bisht, K. S., and Jain, R. (1994). Novel constituents of Uvaria species. Nat. Prod. Rep. 11, 219-224. doi: $10.1039 /$ np9941100219

Pautz, A., Art, J., Hahn, S., Nowag, S., Voss, C., and Kleinert, H. (2010). Regulation of the expression of inducible nitric oxide synthase. Nitric Oxide 23, 75-93. doi: 10.1016/j.niox.2010.04.007

Petersen, L. J., Church, M. K., and Skov, P. S. (1997). Platelet-activating factor induces histamine release from human skin mast cells in vivo, which is reduced by local nerve blockade. J. Aller. Clin. Immunol. 99, 640-647. doi: 10.1016/S0091-6749(97)70026-5

Phillips, O., and Miller, J. S. (2002). Global Patterns of Plant Diversity: Alwyn H. Gentry's Forest Transect Data Set. St. Louis, Mo: Missouri Botanical Press.

Pieme, C. A., Kumar, S. G., Dongmo, M. S., Moukette, B. M., Boyoum, F. F., Ngogang, J. Y., et al. (2014). Antiproliferative activity and induction of apoptosis by Annona muricata (Annonaceae) extract on human cancer cells. BMC Complement. Altern. Med. 14:516. doi: 10.1186/1472-6882-14-516

Pikarsky, E., Porat, R. M., Stein, I., Abramovitch, R., Amit, S., Kasem, S., et al. (2004). NF-kappaB functions as a tumour promoter in inflammationassociated cancer. Nature 431, 461-466. doi: 10.1038/nature02924

Popoola, T. D., Awodele, O., Omisanya, A., Obi, N., Umezinwa, C., and Fatokun, A. A. (2016). Three indigenous plants used in anti-cancer remedies, Garcinia kola Heckel (stem bark), Uvaria chamae P. Beauv. (root) and Olax subscorpioidea Oliv. (root) show analgesic and anti-inflammatory activities in animal models. J. Ethnopharmacol. 194, 440-449. doi: 10.1016/j.jep.2016.09.046

Pourmorad, F., Hosseinimehr, S., and Shahabimajd, N. (2006). Antioxidant activity, phenol and flavonoid contents of some selected Iranian medicinal plants. Afr. J. Biotechnol. 5. 1142-1145.

Queiroz, J. C., Antoniolli, A. R., Quintans-Júnior, L. J., Brito, R. G., Barreto, R. S., Costa, E. V., et al. (2014). Evaluation of the antiinflammatory and antinociceptive effects of the essential oil from leaves of Xylopia laevigata in experimental models. ScientificWorldJournal 2014:816450. doi: 10.1155/2014/816450

Rabelo, S. V., Quintans, L. J., Costa, E. V., da Silva Almeida, J. R. G., and Júnior, L.J.Q. (2016). "Annona species (Annonaceae) oils," in Essential Oils in Food
Preservation, Flavor and Safety, 1st Edn., Chapter 24, ed V. Preedy (Academic Press - Elsevier), 221-229. doi: 10.1016/B978-0-12-416641-7.00024-9

Rahman, M. M., Lopa, S. S., Sadik, G., Islam, R., Khondkar, P., Alam, A. K., et al. (2005). Antibacterial and cytotoxic compounds from the bark of Cananga odorata. Fitoterapia 76, 758-761. doi: 10.1016/j.fitote.2005.08.011

Ravikumar, Y. S., Mahadevan, K. M., Kumaraswamy, M. N., Vaidya, V. P., Manjunatha, H., Kumar, V., et al. (2008). Antioxidant, cytotoxic and genotoxic evaluation of alcoholic extract of Polyalthia cerasoides (Roxb.) Bedd. Environ. Toxicol. Pharmacol. 26, 142-146. doi: 10.1016/j.etap.2008.03.001

Rayanil, K.-,o., Sutassanawichanna, W., Suntornwat, O., and Tuntiwachwuttikul, P. (2016). A new dihydrobenzofuran lignan and potential $\alpha$-glucosidase inhibitory activity of isolated compounds from Mitrephora teysmannii. Nat. Prod. Res. 30, 2675-2681. doi: 10.1080/14786419.2016.1143830

Reuter, S., Gupta, S. C., Chaturvedi, M. M., and Aggarwal, B. B. (2010). Oxidative stress, inflammation, and cancer: how are they linked? Free Radic. Biol. Med. 49, 1603-1616. doi: 10.1016/j.freeradbiomed.2010.09.006

Rocha, R. S., Kassuya, C. A., Formagio, A. S., Mauro Mde, O., Andrade-Silva, M., Monreal, A. C., et al. (2016). Analysis of the anti-inflammatory and chemopreventive potential and description of the antimutagenic mode of action of the Annona crassiflora methanolic extract. Pharm. Biol. 54, 35-47. doi: 10.3109/13880209.2015.1014567

Rojano, B., Perez, E., Figadere, B., Martin, M. T., Recio, M. C., Giner, R., et al. (2007). Constituents of Oxandra cf. xylopioides with anti-inflammatory activity. J. Nat. Prod. 70, 835-838. doi: 10.1021/np060333v

Rouzer, C. A., and Marnett, L. J. (2009). Cyclooxygenases: structural and functional insights. J. Lipid Res. 50, S29-S34. doi: 10.1194/jlr.R800042-JLR200

Saadawi, S., Jalil, J., Jasamai, M., and Jantan, I. (2012). Inhibitory effects of acetylmelodorinol, chrysin and polycarpol from Mitrella kentii on prostaglandin $\mathrm{E}(2)$ and Thromboxane $\mathrm{B}(2)$ production and platelet activating factor receptor binding. Molecules 17, 4824-4835. doi: 10.3390/molecules17054824

Saha, M. R., Jahangir, R., Vhuiyan, M. M. I., and Biva, I. J. (2008). In vitro nitric oxide scavenging activity of ethanol leaf extracts of four Bangladeshi medicinal plants. Stamford J. Pharm. Sci. 1, 57-62. doi: 10.3329/sjps.v1i1.1809

Sahlmann, C.-O., and Ströbel, P. (2016). Pathophysiology of inflammation. Nuklearmedizin 55, 1-6.

Saunders, R. M. (2012). The diversity and evolution of pollination systems in Annonaceae. Bot. J. Linnean Soc. 169, 222-244. doi: 10.1111/j.1095-8339.2011.01208.x

Sauquet, H., Doyle, J. A., Scharaschkin, T., Borsch, T., Hilu, K. W., Chatrou, L. W., et al. (2003). Phylogenetic analysis of Magnoliales and Myristicaceae based on multiple data sets: implications for character evolution. Bot. J. Linnean Soc. 142, 125-186. doi: 10.1046/j.1095-8339.2003.00171.x

Sauquet, H., and Le Thomas, A. (2003). Pollen diversity and evolution in Myristicaceae (Magnoliales). Int. J. Plant Sci. 164, 613-628. doi: 10.1086/375424

Scartezzini, P., and Speroni, E. (2000). Review on some plants of Indian traditional medicine with antioxidant activity. J. Ethnopharmacol. 71, 23-43. doi: 10.1016/S0378-8741(00)00213-0

Seangphakdee, P., Pompimon, W., Meepowpan, P., Panthong, A., Chiranthanut, N., Banjerdpongchai, R., et al. (2013). Anti-inflammatory and anticancer activities of (-)-zeylenol from stems of Uvaria grandiflora. ScienceAsia 39, 610-614. doi: 10.2306/scienceasia1513-1874.2013.39.610

Shih, Y. T., Hsu, Y. Y., Chang, F. R., Wu, Y. C., and Lo, Y. C. (2010). 6-Hydroxycleroda-3,13-dien-15,16-olide protects neuronal cells from lipopolysaccharide-induced neurotoxicity through the inhibition of microgliamediated inflammation. Planta Med. 76, 120-127. doi: 10.1055/s-0029-1186005

Shirwaikar, A., Rajendran, K., and Kumar, C. D. (2004). In vitro antioxidant studies of Annona squamosa Linn. leaves. Indi. J. Exp. Biol. 42, 803-807.

Sidahmed, H. M., Azizan, A. H., Mohan, S., Abdulla, M. A., Abdelwahab, S. I., Taha, M. M., et al. (2013). Gastroprotective effect of desmosdumotin $\mathrm{C}$ isolated from Mitrella kentii against ethanol-induced gastric mucosal hemorrhage in rats: possible involvement of glutathione, heat-shock protein70, sulfhydryl compounds, nitric oxide, and anti-Helicobacter pylori activity. BMC Complement. Altern. Med. 13:183. doi: 10.1186/1472-6882-13-183

Silva, D. B., Tulli, E. C. O., Garcez, W. S., Nascimento, E. A., and Siqueira, J. M. (2007). Chemical constituents of the underground stem bark of Duguetia furfuracea (Annonaceae). J. Braz. Chem. Soc. 18, 1560-1565. doi: 10.1590/S0103-50532007000800017 
Silva, J. C., Araujo Cde, S., de Lima-Saraiva, S. R., de Oliveira-Junior, R. G., Diniz, T. C., Wanderley, C. W., et al. (2015). Antinociceptive and anti-inflammatory activities of the ethanolic extract of Annona vepretorum Mart. (Annonaceae) in rodents. BMC Complement. Altern. Med. 15:197. doi: 10.1186/s12906-015-0716-2

Silva, J. J., Cerdeira, C. D., Chavasco, J. M., Cintra, A. B., Silva, C. B., Mendonça, A. N., et al. (2014). In vitro screening antibacterial activity of Bidens pilosa Linne and Annona crassiflora Mart. against oxacillin resistant Staphylococcus aureus (ORSA) from the aerial environment at the dental clinic. Rev. Instit. Med. Trop. São Paulo 56, 333-340. doi: 10.1590/S0036-46652014000400011

Siqueira, C. A., Serain, A. F., Pascoal, A. C., Andreazza, N. L., de Lourenço, C. C., Ruiz, A. L., et al. (2015). Bioactivity and chemical composition of the essential oil from the leaves of Guatteria australis A.St.-Hil. Nat. Prod. Res. 29, 1966-1969. doi: 10.1080/14786419.2015.1015017

Smith, J. B., and Willis, A. L. (1971). Aspirin selectively inhibits prostaglandin production in human platelets. Nature 231, 235-237. doi: $10.1038 /$ newbio231235a0

Soares, E. R., Silva, F., Almeida, R. A., Lima, B. R., Silva Filho, F. A., Barison, A., et al. (2015). Direct infusion ESI-IT-MSn alkaloid profile and isolation of tetrahydroharman and other alkaloids from Bocageopsis pleiosperma maas (Annonaceae). Phytochem. Anal. 26, 339-345. doi: 10.1002/pca.2568

Solomon-Wisdom, G. O., Ugoh, S. C., and Mohammed, B. (2014). Phytochemical screening and antimicrobial activities of Annona muricata (L) leaf extract. Am. J. Biol. Chem. Pharm. Sci. 2, 1-7.

Soltis, P. S., and Soltis, D. E. (2004). The origin and diversification of angiosperms. Am. J. Bot. 91, 1614-1626. doi: 10.3732/ajb.91.10.1614

Somsrisa, J., Meepowpan, P., Krachodnok, S., Thaisuchat, H., Punyanitya, S., Nantasaen, N., et al. (2013). Dihydrochalcones with antiinflammatory activity from leaves and twigs of cyathostemma argenteum. Molecules 18, 6898-6907. doi: 10.3390/molecules 18066898

Stafforini, D. M., McIntyre, T. M., Zimmerman, G. A., and Prescott, S. M. (2003). Platelet-activating factor, a pleiotrophic mediator of physiological and pathological processes. Crit. Rev. Clin. Lab. Sci. 40, 643-672. doi: $10.1080 / 714037693$

Suzumura, A., Takeuchi, H., Zhang, G., Kuno, R., and Mizuno, T. (2006). Roles of glia-derived cytokines on neuronal degeneration and regeneration. Ann. N.Y. Acad. Sci. 1088, 219-229. doi: 10.1196/annals.1366.012

Taha, H., Arya, A., Paydar, M., Looi, C. Y., Wong, W. F., Vasudeva Murthy, C. R., et al. (2014). Upregulation of insulin secretion and downregulation of pro-inflammatory cytokines, oxidative stress and hyperglycemia in STZnicotinamide-induced type 2 diabetic rats by Pseuduvaria monticola bark extract. Food Chem. Toxicol. 66, 295-306. doi: 10.1016/j.fct.2014.01.054

Takhtajan, A. (2009). Flowering Plants. Springer Science \& Business Media.

Tak, P. P., and Firestein, G. S. (2001). NF-kappaB: a key role in inflammatory diseases. J. Clin. Invest. 107, 7-11. doi: 10.1172/JCI11830

Taniyama, Y., and Griendling, K. K. (2003). Reactive oxygen species in the vasculature molecular and cellular mechanisms. Hypertension 42, 1075-1081. doi: 10.1161/01.HYP.0000100443.09293.4F

Tan, L. T., Lee, L. H., Yin, W. F., Chan, C. K., Abdul Kadir, H., Chan, K. G., et al. (2015). Traditional uses, phytochemistry, and bioactivities of Cananga odorata (Ylang-Ylang). Evid. Based Complement. Altern. Med. 2015:896314. doi: $10.1155 / 2015 / 896314$

Tanna, A., Nair, R., and Chanda, S. (2009). Assessment of anti-inflammatory and hepatoprotective potency of Polyalthia longifolia var. pendula leaf in Wistar albino rats. J. Nat. Med. 63, 80-85. doi: 10.1007/s11418-008-0288-2

Tan, P. V., Nyasse, B., Enow-Orock, G. E., Wafo, P., and Forcha, E. A. (2000). Prophylactic and healing properties of a new anti-ulcer compound from Enantia chlorantha in rats. Phytomedicine 7, 291-296. doi: $10.1016 / 50944-7113(00) 80046-X$

Tan, P. V., Boda, M., Enow-Orock, G. E., Etoa, F.-X., and Bitolog, P. (2007). Acute and sub-acute toxicity profile of the aqueous stem bark extract of Enantia chlorantha Oliver (Annonaceae) in laboratory animals. Pharmacologyonline 1, 304-313.

Thang, T. D., Dai, D. N., Hoi, T. M., and Ogunwande, I. A. (2013a). Chemical compositions of the leaf essential oils of some Annonaceae from Vietnam. J. Essent. Oil Res. 25, 85-91. doi: 10.1080/10412905.2012.755475

Thang, T. D., Kuo, P. C., Huang, G. J., Hung, N. H., Huang, B. S., Yang, M. L., et al. (2013b). Chemical constituents from the leaves of Annona reticulata and their inhibitory effects on NO production. Molecules 18, 4477-4486 doi: 10.3390/molecules 18044477

Thao, N. P., Luyen, B. T., Tai, B. H., Cuong, N. M., Kim, Y. C., Minh, C. V., et al. (2015). Chemical constituents of Miliusa balansae leaves and inhibition of nitric oxide production in lipopolysaccharide-induced RAW 264.7 cells. Bioorg. Med. Chem. Lett. 25, 3859-3863. doi: 10.1016/j.bmcl.2015.07.056

Van Waes, C. (2007). Nuclear factor-кB in development, prevention, and therapy of cancer. Clin. Cancer Res. 13, 1076-1082. doi: 10.1158/1078-0432.CCR-06-2221

Van Wyk, B.-E., and Wink, M. (2004). Medicinal Plants of the World: An Illustrated Scientific Guide to Important Medicinal Plants and Their Uses. Portland: Timber Press.

Vendramini-Costa, D. B., Francescone, R., Posocco, D., Hou, V., Dmitrieva, O., Hensley, H., et al. (2017). Anti-inflammatory natural product goniothalamin reduces colitis-associated and sporadic colorectal tumorigenesis. Carcinogenesis 38, 51-63. doi: 10.1093/carcin/bgw112

Vendramini-Costa, D. B., Monteiro, K. M., Iwamoto, L. H., Jorge, M. P., Tinti, S. V., Pilli, R. A., et al. (2014). Gastroprotective effects of goniothalamin against ethanol and indomethacin-induced gastric lesions in rats: role of prostaglandins, nitric oxide and sulfhydryl compounds. Chem. Biol. Interact. 224C, 206-212. doi: 10.1016/j.cbi.2014.10.025

Vilar, J. B., Ferreira, F. L., Ferri, P. H., Guillo, L. A., and Chen Chen, L. (2008). Assessment of the mutagenic, antimutagenic and cytotoxic activities of ethanolic extract of araticum (Annona crassiflora Mart. 1841) by micronucleus test in mice. Braz. J. Biol. 68, 141-147. doi: 10.1590/S1519-69842008000100020

Villagra, J., Shiva, S., Hunter, L. A., Machado, R. F., Gladwin, M. T., and Kato, G. J. (2007). Platelet activation in patients with sickle disease, hemolysis-associated pulmonary hypertension, and nitric oxide scavenging by cell-free hemoglobin. Blood 110, 2166-2172. doi: 10.1182/blood-2006-12-061697

Watson, L., and Dallwitz, M. (1999). The Families of Flowering Plants: Descriptions, Illustrations, Identification, and Information Retrieval. New Orleans, LA University of New Orleans.

Weber, C., and Noels, H. (2011). Atherosclerosis: current pathogenesis and therapeutic options. Nat. Med. 17, 1410-1422. doi: 10.1038/nm.2538

Whiteley, W., Jackson, C., Lewis, S., Lowe, G., Rumley, A., Sandercock, P., et al. (2009). Inflammatory markers and poor outcome after stroke: a prospective cohort study and systematic review of interleukin-6. PLoS Med. 6:e1000145. doi: 10.1371/journal.pmed.1000145

Wiart, C. (2006). Medicinal Plants of the Asia-Pacific: Drugs for the Future? Malaysia: World Scientific.

Wiart, C. (2007). "Plants for chemotherapy of neoplastic diseases," in Ethnopharmacology of Medicinal Plants (Totowa, NJ: Humana Press), 155-217. doi: 10.1007/978-1-59745-160-4_3

Williams, C. S., Mann, M., and DuBois, R. N. (1999). The role of cyclooxygenases in inflammation, cancer, and development. Oncogene 18, 7908-7916. doi: 10.1038/sj.onc. 1203286

Woguem, V., Fogang, H. P., Maggi, F., Tapondjou, L. A., Womeni, H. M., Quassinti, L., et al. (2014). Volatile oil from striped African pepper (Xylopia parviflora, Annonaceae) possesses notable chemopreventive, antiinflammatory and antimicrobial potential. Food Chem. 149, 183-189. doi: 10.1016/j.foodchem.2013.10.093

Wolfreys, K., and Oliveira, D. B. (1997). Alterations in intracellular reactive oxygen species generation and redox potential modulate mast cell function. Eur. J. Immunol. 27, 297-306. doi: 10.1002/eji.1830270143

Woode, E., Ameyaw, E. O., Boakye-Gyasi, E., and Abotsi, W. K. (2012). Analgesic effects of an ethanol extract of the fruits of Xylopia aethiopica (Dunal) A. Rich (Annonaceae) and the major constituent, xylopic acid in murine models. J. Pharm. Bioall. Sci. 4, 291. doi: 10.4103/0975-7406.103251

Wu, P., Wu, M., Xu, L., Xie, H., and Wei, X. (2014). Anti-inflammatory cyclopeptides from exocarps of sugar-apples. Food Chem. 152, 23-28. doi: 10.1016/j.foodchem.2013.11.100

Wu, T. H., Cheng, Y. Y., Chen, C. J., Ng, L. T., Chou, L. C., Huang, L. J., et al. (2014). Three new clerodane diterpenes from Polyalthia longifolia var. pendula. Molecules 19, 2049-2060. doi: 10.3390/molecules19022049

Wu, T. Y., Yang, I. H., Tsai, Y. T., Wang, J. Y., Shiurba, R., Hsieh, T. J., et al. (2012). Isodesacetyluvaricin, an Annonaceous acetogenin, specifically inhibits gene expression of cyclooxygenase-2. J. Nat. Prod. 75, 572-576. doi: $10.1021 / \mathrm{np} 200719 \mathrm{r}$ 
Yamamoto, Y., and Gaynor, R. B. (2001a). Role of the NF-kappaB pathway in the pathogenesis of human disease states. Curr. Mol. Med. 1, 287-296. doi: $10.2174 / 1566524013363816$

Yamamoto, Y., and Gaynor, R. B. (2001b). Therapeutic potential of inhibition of the NF-kappaB pathway in the treatment of inflammation and cancer. J. Clin. Invest. 107, 135-142. doi: 10.1172/JCI11914

Yang, F., Jove, V., Chang, S., Hedvat, M., Liu, L., Buettner, R., et al. (2012). Bortezomib induces apoptosis and growth suppression in human medulloblastoma cells, associated with inhibition of AKT and NF-?B signaling, and synergizes with an ERK inhibitor. Cancer Biol. Ther. 13, 349. doi: 10.4161/cbt.19239

Yang, R. M., Li, W. M., Hu, W. J., Huang, W. H., Zhu, C. Y., Yu, J. G., et al. (2015). Anticancer effect of total annonaceous acetogenins on hepatocarcinoma. Chin. J. Integr. Med. 21, 682-688. doi: 10.1007/s11655-014-1845-Z

Yang, Y. L., Chang, F. R., Wu, C. C., Wang, W. Y., and Wu, Y. C. (2002). New ent-kaurane diterpenoids with anti-platelet aggregation activity from Annona squamosa. J. Nat. Prod. 65, 1462-1467. doi: 10.1021/np020191e

Yang, Z., Lu, W., Ma, X., and Song, D. (2012). Bioassay-guided isolation of an alkaloid with antiangiogenic and antitumor activities from the extract of Fissistigma cavaleriei root. Phytomedicine 19, 301-305. doi: 10.1016/j.phymed.2011.11.009

Yeh, S. H., Chang, F. R., Wu, Y. C., Yang, Y. L., Zhuo, S. K., and Hwang, T. L. (2005). An anti-inflammatory ent-kaurane from the stems of Annona squamosa that inhibits various human neutrophil functions. Planta Med. 71, 904-909. doi: 10.1055/s-2005-871234

Yeo, D., Dinica, R., Yapi, H. F., Furdui, B., Praisler, M., Djaman, A. J., et al. (2011). [Evaluation of the anti-inflammatory activity and phytochemical screening of Annona senegalensis leaves]. Therapie 66, 73-80. doi: 10.2515/therapie/ 2010076

Yiu, G. K., and Toker, A. (2006). NFAT induces breast cancer cell invasion by promoting the induction of cyclooxygenase-2. J. Biol. Chem. 281, 12210-12217. doi: 10.1074/jbc.M600184200

Zamora, R., Vodovotz, Y., and Billiar, T. R. (2000). Inducible nitric oxide synthase and inflammatory diseases. Mol. Med. 6, 347.
Zhang, J., Li, C., Guo, X., and Wang, G. (2001). Effect of buyang huanwu decoction on platelet activating factor content in arterial blood pre-and post-arterial thrombosis in rats. J. Tradit. Chin. Med. 21, 299-302.

Zhang, J., Zhou, H., Jiang, S., Jin, J., Li, W., Wang, W., et al. (2015). AA092, an annonaceous acetogenin mimetic, attenuates angiogenesis in a mouse model of inflammation-induced corneal neovascularization. Int. Immunopharmacol. 28, 997-1002. doi: 10.1016/j.intimp.2015.08.009

Zhang, N., Hartig, H., Dzhagalov, I., Draper, D., and He, Y. W. (2005). The role of apoptosis in the development and function of T lymphocytes. Cell Res. 15, 749-769. doi: 10.1038/sj.cr.7290345

Zhang, Y., Brovkovych, V., Brovkovych, S., Tan, F., Lee, B. S., Sharma, T. et al. (2007). Dynamic receptor-dependent activation of inducible nitric-oxide synthase by ERK-mediated phosphorylation of Ser745. J. Biol. Chem. 282, 32453-32461. doi: 10.1074/jbc.M706242200

Zhou, R., Yazdi, A. S., Menu, P., and Tschopp, J. (2011). A role for mitochondria in NLRP3 inflammasome activation. Nature 469, 221-225. doi: $10.1038 /$ nature 09663

Ziebell, J. M., and Morganti-Kossmann, M. C. (2010). Involvement of pro-and anti-inflammatory cytokines and chemokines in the pathophysiology of traumatic brain injury. Neurotherapeutics 7, 22-30. doi: 10.1016/j.nurt.2009.10.016

Zomlefer, W. B. (1994). Guide to Flowering Plant Families. Chapel Hill, NC: The University of North Carolina Press.

Conflict of Interest Statement: The authors declare that the research was conducted in the absence of any commercial or financial relationships that could be construed as a potential conflict of interest.

Copyright (c) 2017 Attiq, Jalil and Husain. This is an open-access article distributed under the terms of the Creative Commons Attribution License (CC BY). The use, distribution or reproduction in other forums is permitted, provided the original author(s) or licensor are credited and that the original publication in this journal is cited, in accordance with accepted academic practice. No use, distribution or reproduction is permitted which does not comply with these terms. 